\title{
A New Dislocation-density-function Dynamics Scheme for Computational Crystal Plasticity by Explicit Consideration of Dislocation Elastic Interactions
}

\author{
H.S. Leung, P.S.S. Leung, B. Cheng and A.H.W. Ngan ${ }^{\S}$ \\ Department of Mechanical Engineering, The University of Hong Kong, \\ Pokfulam Road, Hong Kong, P.R. China \\ ${ }^{\S}$ Corresponding author (email: $\underline{\text { hwngan@hku.hk) }}$
}

\begin{abstract}
Current strategies of computational crystal plasticity that focus on individual atoms or dislocations are impractical for real-scale, large-strain problems even with today's computing power. Dislocation-density based approaches are a way forward but a critical issue to address is a realistic description of the interactions between dislocations. In this paper, a new scheme for computational dynamics of dislocation-density functions is proposed, which takes full consideration of the mutual elastic interactions between dislocations based on the Hirth-Lothe formulation. Other features considered include (i) the continuity nature of the movements of dislocation densities, (ii) forest hardening, (iii) generation according to high spatial gradients in dislocation densities, and (iv) annihilation. Numerical implementation by the finite-volume method, which is well suited for flow problems with high gradients, is discussed. Numerical examples performed for a single-crystal aluminum model show typical strength anisotropy behavior comparable to experimental observations. Furthermore, a detailed case study on small-scale crystal plasticity successfully captures a number of key experimental features, including power-law relation between strength and size, low dislocation storage and jerky deformation.
\end{abstract}

Keywords: Crystal plasticity; dislocations; dislocation-density functions; stress-strain behavior; size effect 


\section{Introduction}

The concept of crystal dislocations has been coined for over a century, with a huge knowledge base on how single dislocations behave, or how they interact in individual events, gathered for a wide range of crystalline materials. On the other hand, although crystal plasticity has to take place in units of dislocations, decades of experience have witnessed the impracticalities involved in computing all the participating dislocations, given that they can quickly multiply to very large quantities during plastic deformation. The introduction of atomistic molecular dynamics (MD) simulation in the 1960's has allowed the cores of dislocations to be studied (Vitek, Perrin and Bowen 1970; Vitek 1974). However, despite computer speeds are faster than ever before, and new rare-event sampling and other computational methodologies have been developed, MD simulations are still limited to nano- space and time scales which are far too small compared to most engineering applications of interest, and it is hard to see how such a bottleneck can be circumvented in the future. In the 1990's, discrete dislocation dynamics (DDD) emerged as a dislocation plasticity simulation technique which deals with the evolution of individual dislocation lines according to their laws of motion (Amodeo and Ghoneim 1990; Devincre and Condat 1992; Devincre and Kubin 1997; Bulatov et al. 1998; Bulatov and Cai 2006). In this approach, however, each curved dislocation line is discretized into short linear segments and very expensive calculation is performed on each of these to predict their trajectories according to their very complicated line-tension and mutual interaction effects. Therefore, although promising results have been demonstrated for situations with relative small quantities of dislocations, this method is impractical for higher dislocation quantities due to the amount of computation involved. DDD is therefore self-limiting at increasing strains, and is not practical for situations where patterns of dense dislocations develop (Walgraef and Aifantis 1985; Aifantis 1986; Hähner 1996; Ngan 2005; Pontes, Walgraef and Aifantis 2006), although observations from DDD simulations can be fed into a continuum crystal plasticity model as a multi-scale approach, to predict more realistic behaviors (Devincre, Hoc and Kubin 2008).

In stark contrast with these approaches which emphasize on individual atoms or dislocation segments, a number of researchers have advocated the use of a modelling strategy that focuses on dislocation density (Walgraef and Aifantis 1985; Groma 1997; Acharya 2001; Arsenlis et al. 2004; Zhou and Sun 2004; Evers, Brekelmans and

Geers 2004; Yefimov and Van der Giessen 2005; Pontes, Walgraef and Aifantis 2006; Ma, Roters and Raabe 2006; Hochrainer, Zaiser and Gumbsch 2007; Lee et al. 2010; Watanabe et al., 2010; Alankar, Eisenlohr and Raabe 2011; Hirschberger et al. 2011; Bargmann, Svendsen and Ekh 2011; Liu et al., 2011; Shanthraj and Zikry 2012; 
Engels, Ma and Hartmaier 2012; Aghababaei and Joshi 2013; Li et al. 2013). Unlike DDD which becomes handicapped at high strains, such a strategy would be well suited for large-strain problems with high quantities of dislocations, since any amount of dislocations can still be represented a dislocation density. The earlier group of dislocation-density models deals with discrete categories of dislocation density, such as mobile vs immobile, cell-interior vs cell-wall, edge vs screw, etc. (Roters, Raabe and Gottstein 2000; Prasad, Goerdeler and Gottstein 2005; Ma, Roters and Raabe 2006; Alankar, Eisenlohr and Raabe 2011; Vinogradov, Yasnikov and Estrin 2012), but without considering the field nature of the dislocation densities and the conservative nature of their motion (Walgraef and Aifantis 1985). A more sophisticated group of approach focuses primarily on the kinematics or dynamics of slip systems (Asaro and Rice 1977) and their relationships to densities of dislocations. Typically, the shear of slip systems against a critical resolved shear stress governed by certain basic dislocation-level physics, such as Taylor's forest hardening, is considered (Busso et al. 2000; Dunne et al. 2007; Alankar, Mastorakos and Field 2009; Dunne et al. 2012; Cordero et al. 2012). Other models have focused on dislocation densities as continuous functions of space, with conservation including generation and annihilation duly taken into account (Acharya 2001; Arsenlis et al. 2004; Zhou and Sun 2004; Evers, Brekelmans and Geers 2004; Yefimov and Van der Giessen 2005; Pontes, Walgraef and Aifantis 2006; Hochrainer, Zaiser and Gumbsch 2007; Hirschberger et al. 2011; Bargmann, Svendsen and Ekh 2011; Puri, Das and Acharya 2011). These models are based on crystal kinematics rules which govern the relationship between the evolution of geometrically necessary dislocations (GNDs) and the rate of change of the crystal shape (Asaro and Rice 1977). In doing so, the statistically stored dislocations (SSDs) are modeled much less rigorously, and so the interactions between dislocations and the internal stresses are not completely described.

Still within the framework of crystal kinematics, other models have featured improved descriptions of the internal stresses that resist slip. In the "field dislocation mechanics" (FDM) and "phenomenological mesoscale field dislocation mechanics" (PMFDM) models developed by Acharya and co-workers (Acharya 2001; Taupin et al. 2008; Puri, Das and Acharya 2011), the crystal kinematics laws for the GND evolution are modified to include the effects of lattice incompatibility in the plastic and elastic deformation matrices due to the presence of the dislocations themselves. The evolution of the GNDs is coupled to that of SSDs, the motion of which is modeled in terms of a phenomenological back-stress interaction resistance which involves empirical hardening and recovery coefficients (Puri, Das and Acharya 2011). The models by Yefimov and Giessen (2005) and Hirschberger et al. (2011) also 
involve a similar empirical back stress resistance, which lacks details of the long-range elastic interactions between dislocations. In the model by Arsenlis et al. (2004), a configurational resistance is also involved which is an empirical back stress pertinent only to elastic interactions in 2-D dislocation arrays (Groma et al. 2003). In the recent approach by Bertin, Capolungo and Beyerlein (2013), the coupled evolutions of GNDs and SSDs are also considered, and for the SSDs, a virtual loop concept is involved to sample interactions for different line orientations.

Another line of development to capture realistic dislocation interactions was made by Hochrainer and co-workers (Hochrainer, Zaiser and Gumbsch 2007; Zaiser et al. 2007; Sandfeld et al. 2010; Sandfeld et al. 2011). In their continuum dislocation dynamics (CDD) theory, the curvature and line-tension effects of dislocations are emphasized as the main factors contributing to the internal stress, in addition to Taylor's interactions. The inclusion of the dislocation curvature requires its evolution to be modeled as a coupled problem with the evolution of the dislocation density, and this inevitably greatly increases the computational efforts needed (Sandfeld et al. 2011). For this reason, successful numerical implementation has been limited to situations involving crude discretization of the line-direction space (Zaiser et al. 2007), or very simple slip-system configurations (Zaiser et al. 2007; Sandfeld et al. 2010; Sandfeld et al. 2011). While this approach is elegant, efficient numerical implementation algorithms are yet to be developed for problems involving realistic dislocation microstructures. Moreover, this approach highlights the "self" line tension and Taylor hardening as the only internal stresses (Zaiser et al. 2007; Sandfeld et al. 2011), while the long-range elastic interactions between dislocation groups are not modelled.

The above summary points to the fact that an accurate description of the mutual elastic interactions between dislocations has been a critical issue in developing realistic dislocation-density models. In this paper, we propose a new formulation which fully considers such mutual elastic interactions between 3-D dislocation densities, in an exact manner without involving any ad hoc back-stress assumption as in some of the previous models. This is made possible by generalizing the elastic interactions between dislocation segments (Hirth and Lothe 1992) for dislocation densities, and reducing the line-integral formulation involved into an algebraic form comprising only elementary functions which are straightforward enough for efficient numerical implementation. Also, instead of separating the dislocation population into GNDs and SSDs as in the previous crystal-kinematics-based models, we use a fully dynamics approach which is similar to that used in MD and DDD simulations, namely, we calculate the net forces acting on each type of dislocation density situated at each pixel, due to the applied stress net of Taylor and lattice resistances and the elastic 
interactions from other dislocation densities, and then calculate the evolution of the density via a dynamics law. In this paradigm, the evolution of all mobile dislocations, whether GNDs or SSDs, is governed by the same law, as should be the case in reality. To make clear the fact that full dynamics for dislocation-density functions are considered here, we term our approach dislocation-density function dynamics (DDFD) hereafter. For numerical implementation, to cater for high gradients in the dislocation densities, we use the finite volume method (FVM), which is more suitable for fluid-like transport problems with sharp gradients.

\section{General Formulation of Model}

In this section, we will formulate the basic equations of the evolution rules in our model. As in the previous density-based models, we imagine that we cannot see individual dislocation lines, but just densities of them, measured as line length of dislocations per unit volume. The graining of the spatial domain under discussion here is chosen so that it is coarse enough for a material pixel to contain sufficient dislocations for their density to be treated as uniform inside the pixel, but is fine enough compared to the microstructure length scale over which the dislocation density typically varies.

Let $\rho_{\alpha}(\vec{r}, t)$ be the density (length per unit volume) of dislocations of type $\alpha$ at position $\vec{r}$ and time $t$, where $\alpha$ is specified by the following factors:

(i) the slip system $\{\hat{n}, \vec{b}\}$ with $\hat{n}$ being the slip plane unit normal and $\vec{b}$ the Burgers vector,

(ii) the dislocation character (edge, screw or any mixed character), and

(iii) the sign of the dislocation (either + or - ).

In the following, we develop the equation governing the evolution of $\rho_{\alpha}(\vec{r}, t)$, using as much known physics of dislocations as is possible.

\subsection{Dislocation Flux and Strain Rate}

The dislocation flux $\overrightarrow{\mathrm{J}}_{\alpha}(\vec{r}, t)$ is defined as the quantity (line length) of dislocations of type $\alpha$ moving across a perpendicular surface of unit area per unit time. The flux is related to the velocity $\vec{v}_{\alpha}$ of the dislocations by (Walgraef and Aifantis 1985)

$$
\overrightarrow{\mathrm{J}}_{\alpha}(\vec{r}, t)=\rho_{\alpha}(\vec{r}, t) \overrightarrow{\mathrm{v}}_{\alpha}(\vec{r}, t)
$$

The dislocation velocity $\overrightarrow{\mathrm{v}}_{\alpha}(\vec{r}, t)$ is assumed to be a power law of the effective stress $\tau_{\alpha}^{e f f}$ acting on the dislocation (Johnston and Gilman 1959), viz. 


$$
\overrightarrow{\mathrm{v}}_{\alpha}(\vec{r}, t)=\operatorname{sgn}\left(\tau_{\alpha}^{e f f}(\vec{r}, t)\right)(\hat{n} \times \hat{\xi}) v_{0}\left|\tau_{\alpha}^{e f f}(\vec{r}, t) / \tau_{0}\right|^{m}
$$

where $v_{0}, \tau_{0}$ and $m$ are constants, $\operatorname{sgn}(x)$ is the sign function, $\hat{\xi}$ is the unit vector along the dislocation line corresponding to the dislocation type $\alpha$. Details of the effective stress $\tau_{\alpha}^{\text {eff }}$ are given in Section 2.2 below.

Following Walgraef and Aifantis (1985), the evolution of the density of a given dislocation type $\alpha$ is governed by the conservative law:

$$
\dot{\rho}_{\alpha}(\vec{r}, t)=-\vec{\nabla} \cdot \vec{J}_{\alpha}(\vec{r}, t)+\text { net production rate, }
$$

or

$$
\dot{\rho}_{\alpha}(\vec{r}, t)=-\vec{\nabla} \cdot\left[\rho_{\alpha}(\vec{r}, t) \overrightarrow{\mathrm{v}}_{\alpha}(\vec{r}, t)\right]+\text { net production rate }
$$

The net production rate here accounts for dislocation generation and annihilation, and details of these are given in Section 2.3 below.

The Orowan strain-rate field (Orowan 1940) produced by dislocation type $\alpha$ at time $t$ is $\rho_{\alpha}(\vec{r}, t)\left[\overrightarrow{\mathrm{v}}_{\alpha}(\vec{r}, t) \cdot(\hat{\mathrm{n}} \times \hat{\xi})\right](\vec{b} \otimes \hat{\mathrm{n}})=\left|\overrightarrow{\mathrm{J}}_{\alpha}(\vec{r}, t)\right|(\vec{b} \otimes \hat{\mathrm{n}})$, where $\vec{b} \otimes \hat{\mathrm{n}}$ is the tensor product between the two vectors, and so summing the contributions from all dislocation types, the gross plastic strain-rate tensor field is

$$
\dot{\boldsymbol{\varepsilon}}(\vec{r}, t)=\sum_{\alpha}(\vec{b} \otimes \hat{\mathrm{n}})\left[\overrightarrow{\mathrm{J}}_{\alpha}(\vec{r}, t) \cdot(\hat{\mathrm{n}} \times \hat{\xi})\right]
$$

The cumulative plastic strain tensor field is therefore obtainable as

$$
\boldsymbol{\varepsilon}^{p}(\vec{r}, t)=\int_{0}^{t} \dot{\varepsilon}\left(\vec{r}, t^{\prime}\right) d t^{\prime}
$$

As will be discussed later in Section 5, the Orowan strain here represents a pure-shear plastic strain produced by the glide motion of the dislocations at a coarse-grained level, while elastic deformation and the self-fields of the dislocations are not included. It may appear, because of the summation in eqn. (4), that only the glide motions of the GNDs contribute to the Orowan strain rate, while those of the SSDs effectively cancel out. However, the dynamics of the SSDs intricately affect those of the GNDs, and for this reason, the evolution of all dislocations, including both GNDs and SSDs, needs to be considered fully.

\subsection{Effective Glide Stress on Dislocation Systems}

The effective glide stress on the dislocation system in eqn. (2) above is 


$$
\tau_{\alpha}^{\text {eff }}(\vec{r}, t)=\tau_{\alpha}^{\text {ext }}(\vec{r}, t)-\tau_{\alpha}^{f}-\tau_{\alpha}^{\text {Taylor }}+\tau_{\alpha}^{\text {int }}(\vec{r}, t)
$$

where the four terms on the right side are, respectively,

(i) the externally applied stress $\tau_{\alpha}^{e x t}$, which is the component of the applied stress on the slip plane (with normal $\hat{n}$ ) along the $\hat{n} \times \hat{\xi}$ direction;

(ii) the lattice friction $\tau_{\alpha}^{f}$ which is in general a function of the dislocation type $\alpha$, but in a simplified exercise, can be supposed to be a constant $\tau^{f}$ independent of $\alpha$;

(iii) the Taylor hardening resistance $\tau_{\alpha}^{\text {Taylor }}$ due to the short-ranged interactions between dislocations; and

(iv) the long-range elastic interaction stress $\tau_{\alpha}^{\text {int }}$ with all other dislocations in the system, in addition to the Taylor interaction mechanism.

\subsubsection{Long-range elastic interaction stress $\tau_{\alpha}^{i n t}$}

As discussed by Hirth and Lothe (1992), and more recently by Balluffi (2012), the elastic interaction force between two infinitesimally short dislocation segments 1 and 2 as shown in Fig. 1(a) is

$$
\begin{gathered}
\delta \vec{f}=-\frac{\mu}{8 \pi}\left[\left(\vec{b}_{2} \times \vec{b}_{1}\right) \cdot \vec{\nabla}\left(\nabla^{2} \mathrm{R}\right)\right]\left(d \vec{l}_{1} \times d \vec{l}_{2}\right) \\
-\frac{\mu}{8 \pi}\left\{\left[\vec{b}_{2} \times \vec{\nabla}\left(\nabla^{2} \mathrm{R}\right)\right] \times d \vec{l}_{1}\right\}\left(\vec{b}_{1} \cdot d \vec{l}_{2}\right) \\
-\frac{\mu}{4 \pi(1-v)}\left[\left(\vec{b}_{2} \times d \vec{l}_{2}\right) \cdot \vec{\nabla}\right]\left(d \vec{l}_{1} \times \vec{b}_{1} \mathrm{~T}\right) \\
+\frac{\mu}{4 \pi(1-v)}\left[\left(\vec{b}_{2} \times d \vec{l}_{2}\right) \cdot \vec{\nabla}\left(\nabla^{2} \mathrm{R}\right)\right]\left(d \vec{l}_{1} \times \vec{b}_{1}\right)
\end{gathered}
$$

where $R$ is the distance between the two segments, $\vec{\nabla}=\hat{R} \frac{d}{d R}$ for operation on any function which depends on $R$ alone, and $\mathbb{T}$ is the tensor

$$
\mathbb{T}=\frac{\partial^{2} R}{\partial x_{a} \partial x_{b}} \hat{e}_{a} \otimes \hat{e}_{b}
$$

with $x_{a}$ and $x_{b}$ being the relative coordinates of dislocation segment 1 with respect to segment 2 .

In our DDFD model, we generalize eqn. (7) to the situation when two densities of dislocations, instead of two line segments, interact. As shown in Fig. 1(b), the force 
on unit length of dislocation of type $\alpha$ situated at $\vec{r}$, due to a density of dislocations of type $\alpha^{\prime}$ inside an infinitesimal volume $d^{3} r^{\prime}$ at $\vec{r}^{\prime}$, is obtainable from eqn. (7) by letting $d \vec{l}_{1}=\hat{\xi}$ and $d \vec{l}_{2}=\hat{\xi}^{\prime} \rho_{\alpha^{\prime}}\left(\vec{r}^{\prime}, t\right) d^{3} r^{\prime}$, where $\hat{\xi}$ and $\hat{\xi}^{\prime}$ are the unit vectors of the dislocation line corresponding to the two dislocation types at $\vec{r}$ and $\vec{r}^{\prime}$ respectively. Hence, the dislocation-dislocation interaction force on unit length of dislocation, of a general type $\alpha$ at a general position $\vec{r}$, is obtained by integrating the effects of all the dislocations in the system as

$$
\begin{gathered}
\vec{f}_{\alpha}(\vec{r}, t)=-\frac{\mu}{8 \pi} \sum_{\alpha \prime} \iiint \rho_{\alpha^{\prime}}\left(\vec{r}^{\prime}, t\right)\left[\left(\vec{b}^{\prime} \times \vec{b}\right) \cdot \vec{\nabla}\left(\nabla^{2} \mathrm{R}\right)\right]\left(\hat{\xi} \times \hat{\xi}^{\prime}\right) d^{3} r^{\prime} \\
-\frac{\mu}{8 \pi} \sum_{\alpha^{\prime}} \iiint \rho_{\alpha^{\prime}}\left(\vec{r}^{\prime}, t\right)\left\{\left[\vec{b}^{\prime} \times \vec{\nabla}\left(\nabla^{2} \mathrm{R}\right)\right] \times \hat{\xi}\right\}\left(\vec{b} \cdot \hat{\xi}^{\prime}\right) d^{3} r^{\prime} \\
-\frac{\mu}{4 \pi(1-v)} \sum_{\alpha \prime} \iiint \rho_{\alpha^{\prime}}\left(\vec{r}^{\prime}, t\right)\left[\left(\vec{b}^{\prime} \times \hat{\xi}^{\prime}\right) \cdot \vec{\nabla}\right](\hat{\xi} \times \vec{b} \mathbb{T}) d^{3} r^{\prime} \\
+\frac{\mu}{4 \pi(1-v)}(\hat{\xi} \times \vec{b}) \sum_{\alpha^{\prime}} \iiint \rho_{\alpha^{\prime}}\left(\vec{r}^{\prime}, t\right)\left[\left(\vec{b}^{\prime} \times \hat{\xi}^{\prime}\right) \cdot \vec{\nabla}\left(\nabla^{2} \mathrm{R}\right)\right] d^{3} r^{\prime}
\end{gathered}
$$

where $R=\left|\vec{r}-\vec{r}^{\prime}\right|$. The glide component of $\vec{f}_{\alpha}$ is $\vec{f}_{\alpha} \cdot(\hat{n} \times \hat{\xi})$, and so the Peach-Koehler glide stress due to dislocation-dislocation interaction is $\tau_{\alpha}^{\text {int }}=\vec{f}_{\alpha}$. $(\hat{\xi} \times \hat{n}) / b$, i.e.

$$
\begin{gathered}
\tau_{\alpha}^{i n t}(\vec{r}, t)=-\frac{\mu}{8 \pi}(\hat{n} \times \hat{\xi}) \cdot \sum_{\alpha \prime} \iiint \rho_{\alpha^{\prime}}\left(\vec{r}^{\prime}, t\right)\left[\left(\vec{b}^{\prime} \times \hat{b}\right) \cdot \vec{\nabla}\left(\nabla^{2} \mathrm{R}\right)\right]\left(\hat{\xi} \times \hat{\xi}^{\prime}\right) d^{3} r^{\prime} \\
-\frac{\mu}{8 \pi}(\hat{n} \times \hat{\xi}) \cdot \sum_{\alpha \prime} \iiint \rho_{\alpha^{\prime}}\left(\vec{r}^{\prime}, t\right)\left\{\left[\vec{b}^{\prime} \times \vec{\nabla}\left(\nabla^{2} \mathrm{R}\right)\right] \times \hat{\xi}\right\}\left(\hat{b} \cdot \hat{\xi}^{\prime}\right) d^{3} r^{\prime} \\
-\frac{\mu}{4 \pi(1-v)}(\hat{n} \times \hat{\xi}) \cdot \sum_{\alpha^{\prime}} \iiint \rho_{\alpha^{\prime}}\left(\vec{r}^{\prime}, t\right)\left[\left(\vec{b}^{\prime} \times \hat{\xi}^{\prime}\right) \cdot \vec{\nabla}\right](\hat{\xi} \times \hat{b} \mathbb{T}) d^{3} r^{\prime}
\end{gathered}
$$

where $\hat{b}=\vec{b} / b$. Note that the last term in eqn. (8) is the climb force, and so its dot product with $(\hat{n} \times \hat{\xi})$ is zero, and that is why this term is no longer present in eqn. (9). Climb of the dislocations can be considered by adding a term in the direction of $(\hat{\xi} \times \hat{b})$ in the expression for $\overrightarrow{\mathrm{v}}_{\alpha}(\vec{r}, t)$ in eqn. (2), and by considering its dependence on the climb force (the last term in eqn. (8)), but in the present work, this is ignored. Eqn. (9) may be rewritten in a more condensed form as

$$
\tau_{\alpha}^{i n t}(\vec{r}, t)=\sum_{\alpha^{\prime}} \iiint g(\vec{R}, t) \rho_{\alpha^{\prime}}\left(\vec{r}^{\prime}, t\right) d^{3} r^{\prime}
$$


where

$$
\begin{gathered}
g(\vec{R}, t)=-\frac{\mu}{8 \pi}(\hat{n} \times \hat{\xi}) \cdot\left[\left(\vec{b}^{\prime} \times \hat{b}\right) \cdot \vec{\nabla}\left(\nabla^{2} R\right)\right]\left(\hat{\xi} \times \hat{\xi}^{\prime}\right) \\
-\frac{\mu}{8 \pi}(\hat{n} \times \hat{\xi}) \cdot\left\{\left[\vec{b}^{\prime} \times \vec{\nabla}\left(\nabla^{2} R\right)\right] \times \hat{\xi}\right\}\left(\hat{b} \cdot \hat{\xi}^{\prime}\right) \\
-\frac{\mu}{4 \pi(1-v)}(\hat{n} \times \hat{\xi}) \cdot\left[\left(\vec{b}^{\prime} \times \hat{\xi}^{\prime}\right) \cdot \vec{\nabla}\right](\hat{\xi} \times \hat{b} \mathbb{T})
\end{gathered}
$$

and $\vec{R}=\vec{r}-\vec{r}^{\prime}$.

It is worth noting here that, by virtue of the outer summation over all the dislocation types $\alpha^{\prime}$ in eqn. (10), the density $\rho_{\alpha^{\prime}}\left(\vec{r}^{\prime}, t\right)$ at the source location $\vec{r}^{\prime}$ needed to be considered in the integration can simply be replaced by the net GND density, since the effects of all SSDs at $\vec{r}^{\prime}$ sum up to zero. However, the calculated $\tau_{\alpha}^{i n t}(\vec{r}, t)$ at the field point $\vec{r}$ applies to the corresponding dislocation type $\alpha$ there, no matter whether it is GND or SSD. The dynamics of GNDs at the field point $\vec{r}$ are affected by the presence of SSDs in the neighborhood and vice versa, and in the present approach, the dynamics of all dislocations in the structure are considered, without the need to know which are GNDs and which are SSDs.

\subsubsection{Taylor hardening resistance $\tau_{\alpha}^{\text {Taylor }}$}

The coarse-grained, or representative volume element (RVE), approach in DDFD models is pertinent to recent advances in mechanical characterization of micron-sized specimens, which, apart from free-surface effects, may be regarded as the RVEs sampled from a master dislocation structure (Gu and Ngan 2013). Recent experiments on such micro-specimens with their dislocation contents purposely varied have shown that their strength is remarkably well correlated to the square-root of the dislocation density confined in them (Gu and Ngan 2012), thus indicating that Taylor hardening (Taylor 1934) is an important factor of the flow resistance. Franciosi and Zaoui (1982) proposed the following strain-hardening law for fcc crystals

$$
\tau_{\alpha}^{\text {Taylor }}=\mu b \sqrt{\sum_{\alpha \prime} a_{\alpha \alpha^{\prime}} \rho_{\alpha^{\prime}}}
$$

where the interaction coefficients $a_{\alpha \alpha^{\prime}}$ adopt one of six possible values corresponding to self hardening as well as interactions due to coplanar systems, collinear but not coplanar systems, Hirth locks, glissile junctions and Lomer-Cottrell locks. These interaction coefficients, however, are not known a priori, and instead of engaging in a multi-variable fitting exercise as in, for example, Arsenlis and Parks 
(2002), we assume the following reduced scheme in this work:

$$
\begin{array}{lc}
a_{\alpha \alpha^{\prime}}=\left(\alpha_{\perp} \vec{n}_{\alpha} \cdot \vec{\xi}_{\alpha^{\prime}}\right)^{2} & \text { if }\left(\vec{n}_{\alpha} \cdot \vec{\xi}_{\alpha^{\prime}}\right)^{2} \neq 0, \text { and } \\
a_{\alpha \alpha \prime}=a_{\|} & \text {otherwise, }
\end{array}
$$

where $\alpha_{\perp}$ and $a_{\|}$are constant parameters. Eqn. (13) here represents the short-range interactions due to dislocations lying on slip planes intersecting that of type $\alpha$, while eqn. (14) represents short-ranged interactions with dislocations lying on the same slip plane as type $\alpha$. It should be noted that Kubin, Devincre and Hoc (2008) have also proposed corrections to the Taylor hardening based on long-range dislocation interactions, but since the latter are already modeled separately, such corrections are not used in eqn. (13).

\section{3. Dislocation Density Production and Annihilation}

\subsubsection{Dislocation generation}

An important way by which new dislocation lengths are generated arises from the dislocation motion itself. The amount of dislocations of type $\alpha$ may change whenever dislocations of another character $\alpha^{\prime}$ on the same slip plane develop velocity gradients along their own line direction, viz.

$$
\dot{\rho}_{\alpha}^{g e n}=\sum_{\alpha^{\prime} \rightarrow \alpha} \partial\left(\rho_{\alpha^{\prime}} v_{\alpha^{\prime}}\right) / \partial \xi_{\alpha \prime}
$$

To see this, we follow a framework similar to that proposed by Arsenlis et al. (2004), where the dislocation character is limited to only the edge $(e)$ or screw $(s)$ type as shown in Fig. 2. With such an idealization, dislocations of a given slip system $\{\hat{n}, \vec{b}\}$ can only adopt one of the two orthogonal line orientations corresponding to $e$ or $s$, and with the two signs + or - , there will be 4 dislocation types $e+, e-, s+$ and $s-$ on the slip system. Dislocation generation in this case may arise as a rectangular loop expands (Fig. 3(a)), or as a dislocation line bows out between pinned ends (Fig. 3(b)). These two processes can be generalized into a similar concept of dislocations trailing behind - when a dislocation glides, it will produce new trailing dislocations; for example, a gliding edge dislocation will generate new screw lengths (Fig. 3(c)), and vice versa. A special form of eqn. (15) for this case has been given by Arsenlis et al. (2004) as:

$$
\dot{\rho}_{e+}^{g e n}=\dot{\rho}_{e-}^{g e n}=\rho_{s-} \times \frac{v_{s-}}{\ell_{\alpha}}+\rho_{s+} \times \frac{v_{s+}}{\ell_{\alpha}}
$$




$$
\dot{\rho}_{s+}^{g e n}=\dot{\rho}_{s-}^{g e n}=\rho_{e-} \times \frac{v_{e-}}{\ell_{\alpha}}+\rho_{e+} \times \frac{v_{e+}}{\ell_{\alpha}},
$$

where $\ell_{\alpha}$ is the free dislocation length between pinning points, and $v_{e+}$ etc. are magnitudes of dislocation velocities. Eqns. (15) and (16) can also capture Frank-Read sources, which are free segments of dislocations terminated within the lattice at pinning points, and therefore correspond to a step increase of dislocation density in the pixel containing them. In Section 3.3, we will discuss how the criterion in eqn. (15) or (16) for dislocation generation can be implemented numerically.

Apart from trailing dislocations, cross slip of screw dislocations also leads to production of new dislocations, as shown in Fig. 4. Here, the red segment is the cross-slipping segment due to a more favorable stress on an intersecting slip plane, and once it has cross-slipped, the segment disappears in the original system, leading to a reduction of $\rho_{s_{-}}$in the original (111) plane and an increase of density by the same amount in the (1 $\overline{1} \overline{1})$ plane. Alankar, Field and Zbib (2012) have recently discussed the incorporation of cross slip into a DDFD model, by involving a probability function that represents the effects of a cross-slip length, stacking fault energy and the resolved shear stress on the original and cross-slip planes.

\subsubsection{Dipole annihilation}

As considered by Arsenlis et al. (2004), annihilation of dislocation densities occurs whenever opposite signed densities come within a critical capture radius. In the edge-screw idealization, the annihilation rates are given as

$$
\begin{gathered}
\dot{\rho}_{e+}^{a n n}=\dot{\rho}_{e^{-}}^{a n n}=-\rho_{e+} \rho_{e-} R_{e}\left|v_{e+}-v_{e-}\right| \\
\dot{\rho}_{s+}^{a n n}=\dot{\rho}_{s-}^{a n n}=-\rho_{s+} \rho_{s-} R_{s}\left|v_{s+}-v_{s-}\right|
\end{gathered}
$$

where $R_{e}$ and $R_{s}$ are the critical capture radii for edge and screw characters respectively.

\section{Numerical Implementation}

To illustrate the use of the above model, we have performed numerical simulation for an idealized single-crystal fcc model, which consists of 12 slip systems

of the type $\{111\}\left\langle\frac{1}{2}, \frac{1}{2}, 0\right\rangle$. On each slip system, the edge-screw idealization shown in Fig. 2 is used, thus there are 4 dislocation types $e+, e-, s+$ and $s-$ on each slip system. As a result, there are totally 48 types of dislocation densities under consideration. 
As mentioned before, numerical implementation is based on the finite volume method (FVM), for its capability to capture high gradients. FVM is widely used in Computation Fluid Dynamics (Wesseling 2001; Zikanov 2010), and this is also a good methodology for DDFD because dislocation density dynamics are very similar to fluid flow. In particular, for DDFD, the internal states are the dislocation densities $\rho_{\alpha}$ which can flow freely and may exhibit high gradients.

To numerically implement the model framework above using FVM, the equations involved need to be re-cast in discrete forms, and sometimes they need to be modified to suit the FVM context. These are discussed in detail below.

\subsection{Flux}

The main concept in FVM is the discretization of space into cells, and for a typical flux operator

$$
\dot{\rho}+\vec{\nabla} \cdot(\rho \vec{v})
$$

which is pertinent to eqn. (3) in our model, the first-order "upwind" discretization of the $i$-th cell will be

$$
\frac{d \rho_{i}}{d t}+\frac{1}{\Delta x_{i}}\left[(\rho v)_{i+1}-(\rho v)_{i}\right]+\frac{1}{\Delta y_{i}}\left[(\rho v)_{i+1}-(\rho v)_{i}\right]+\frac{1}{\Delta z_{i}}\left[(\rho v)_{i+1}-(\rho v)_{i}\right] .
$$

This is the basic FVM formulation, but in order to handle high gradients, a total variation diminishing scheme must be introduced, and usually Monotone Upstream-centered Schemes for Conservation Laws (MUSCL) (Wesseling 2001) are employed. Here, we used the Kurganov-Tadmor central scheme, which is a second-order, high-resolution MUSCL construction. With this, the flux operator above is re-cast into the semi-discrete form (Wesseling 2001)

$$
\frac{d \rho_{i}}{d t}+\frac{1}{\Delta x_{i}}\left[(\rho v)^{*}{ }_{i+1 / 2}-(\rho v)^{*}{ }_{i-1 / 2}\right]+\cdots
$$

where

$$
\begin{aligned}
& (\rho v)^{*}{ }_{i \pm 1 / 2}=\frac{1}{2}\left\{\left[\rho_{i \pm 1 / 2}^{R} v_{i \pm 1 / 2}+\rho_{i \pm 1 / 2}^{L} v_{i \pm 1 / 2}\right]-\max \left[\left|v_{i}\right|,\left|v_{i \pm 1}\right|\right]\left[\rho_{i \pm 1 / 2}^{R}-\rho_{i \pm 1 / 2}^{L}\right]\right\} \\
& \rho_{i+1 / 2}^{L}=\rho_{i}+0.5 \phi\left(r_{i}\right)\left(\rho_{i+1}-\rho_{i}\right) ; \quad \rho_{i+1 / 2}^{R}=\rho_{i+1}+0.5 \phi\left(r_{i+1}\right)\left(\rho_{i+2}-\rho_{i+1}\right) ; \\
& \rho_{i-1 / 2}^{L}=\rho_{i-1}+0.5 \phi\left(r_{i-1}\right)\left(\rho_{i}-\rho_{i-1}\right) ; \quad \rho_{i-1 / 2}^{R}=\rho_{i}+0.5 \phi\left(r_{i}\right)\left(\rho_{i+1}-\rho_{i}\right) ; \\
& r_{i}=\left(v_{i}-v_{i-1}\right) /\left(v_{i+1}-v_{i}\right) .
\end{aligned}
$$

Here, $\phi(r)$ is the flux limiter, which is a function such that for $r<0, \phi=0$ and 
$\phi=1$ for $r=1$. The main idea of MUCSL is to use slope limited left and right extrapolated states for the cell's approximation, and the left and right states are those at the cell walls, i.e. $i= \pm 1 / 2$. Also MUSCL is combined with the Runge-Kutta time marching method to obtain accurate results.

\subsection{Interaction Stress}

The elastic interaction stress between dislocation densities is given by eqn. (10), and with the edge-screw idealization shown in Fig. 2, the interaction function $g(\vec{R})$ concerned is given in the Appendix as eqn. (A4). As there are 48 types of dislocation densities, there will be $48 \times 48$ pairs of dislocation densities to interact, and by converting eqn. (10) into a discretized form, the total glide stress acting on density type $\alpha$ in the $i$-th cell will be

$$
\tau_{\alpha}^{i n t}\left(\vec{r}_{i}\right)=\sum_{\alpha^{\prime}=1}^{48} \sum_{j \neq i} g\left(\vec{r}_{j}-\vec{r}_{i}\right) \rho_{\alpha^{\prime}}\left(\vec{r}_{j}\right) V
$$

where $j$ are the cells surrounding the $i$-th cell within a cutoff radius $R_{c}$, and $V$ is the cell volume. A cutoff radius $R_{c}$ is used here to increase the computational speed as the interaction force varies with $1 / R^{2}$, and so dislocation densities situated far away can be neglected. In the results presented below in Section $4, R_{c}$ was set to be about half the mean dimension of the size of the specimen.

For the effective stress in eqn. (6), the lattice friction $\tau_{\alpha}^{f}$ is set to be the Peierls-Nabarro stress in the numerical examples given below, i.e.

$$
\tau_{\alpha}^{f}=\frac{2 \mu}{(1-v)} e^{-2 \pi a(1-v) / b},
$$

where $a$ is the interplanar spacing, $b$ is the Burgers vector and $v$ the Poisson ratio.

The Taylor hardening $\tau_{\alpha}^{\text {Taylor }}$ is calculated using eqns. (12) to (14). In the velocity law in eqn. (2), the stress exponent $m$ is set to be 5. Strain rate and strain are calculated using eqns. (4) and (5) respectively.

\subsection{Generation}

As discussed in Section 2.3.1, consideration of dislocation generation is based on finding locations where dislocation densities exhibit high spatial gradients according to eqn. (15). In the FVM scheme when space is discretized into cells of size $\Delta x$, instead of using a global configurational length $\ell_{\alpha}$ we take the units for the consideration of dislocation generation to be the FVM cells, i.e. in eqn. (16) we set $\ell_{\alpha}=\Delta x$, where $\Delta x$ is the cell size. Potential dislocation generation is then 
considered at the walls of the cells, as shown in Fig. 5(a). Here, a dislocation segment moving to the left (the black segment) potentially drags out new dislocation lengths of another orientation (green and blue) at the upper and lower walls of the cell, but whether such new lengths are counted as real generation depends on the criterion set by eqn. (15).

To implement eqn. (15) or (16), if the densities of dislocations of the same type on two adjacent cells are similar (Fig. 5), i.e. $(\rho v / \Delta x)^{i}=(\rho v / \Delta x)^{i+1}$, then the dislocations concerned are considered to be simply threading through the two cells $i$ and $i+1$, and so their movement along the $\hat{n} \times \hat{\xi}$ direction will not lead to generation of new dislocations. This step ensures that the consideration of dislocation generation is not affected by the cell size. On the other hand, if $(\rho v / \Delta x)^{i} \neq$ $(\rho v / \Delta x)^{i+1}$, then the wall between the $i^{t h}$ and $(i+1)^{t h}$ cells will act as a source of the types shown in Fig. 3(a,b), and generation of new dislocation densities will occur. The quantity of new dislocation production at the cell wall thus depends on the difference in $\rho v / \Delta x$ between the two cells, and so eqn. (15) or (16) is recast into:

$$
\begin{aligned}
& \dot{\rho}_{e+}^{i, i+1}=F\left[\left(\frac{\rho v}{\Delta x}\right)_{s+}^{i+1}-\left(\frac{\rho v}{\Delta x}\right)_{s+}^{i}\right]+F\left[\left(\frac{\rho v}{\Delta x}\right)_{s-}^{i+1}-\left(\frac{\rho v}{\Delta x}\right)_{s-}^{i}\right] \\
& \dot{\rho}_{e-}^{i, i+1}=F\left[\left(\frac{\rho v}{\Delta x}\right)_{s+}^{i}-\left(\frac{\rho v}{\Delta x}\right)_{s+}^{i+1}\right]+F\left[\left(\frac{\rho v}{\Delta x}\right)_{s-}^{i}-\left(\frac{\rho v}{\Delta x}\right)_{s-}^{i+1}\right] \\
& \text { etc., }
\end{aligned}
$$

where $F(x)$ is the ramp function (i.e. $F(x)=x$ if $x>0$, and 0 otherwise). However, not all dislocations in cell $i$ are connected to the neighboring cell, and so eqn. (20) would overestimate the new dislocation production. In fact, whenever $(\rho v)^{i} \neq$ $(\rho v)^{i+1}$, the quantity of dislocations generated in cell $i$ is limited by the work done by the net effective stress acting on the cell. Since the power density for a given slip system $\alpha$ is $\rho_{\alpha} v_{\alpha} b \tau_{\alpha}^{e f f}$, the generation rates in eqn. (20) are scaled as follows:

$$
\begin{gathered}
\dot{\rho}_{e+}^{i, i+n} \rightarrow \frac{\dot{\rho}_{e+}^{i, i+n}}{E_{e}\left(\sum_{n} \dot{\rho}_{e+}^{i, i+n}\right)} \times \rho_{e+} v_{e+} b \tau_{e+}^{e f f} ; \\
\dot{\rho}_{e-}^{i, i+n} \rightarrow \frac{\dot{\rho}_{e-}^{i, i+n}}{E_{e}\left(\sum_{n} \dot{\rho}_{e-}^{i, i+n}\right)} \times \rho_{e-} v_{e-} b \tau_{e-}^{e f f} ;
\end{gathered}
$$

where $(i+n)$ denotes the neighbor cells of $i$, and $E_{e}$ is the energy per unit length of 
dislocation. The generated densities at the cell wall are partitioned equally into the two cells, i.e.

$$
\dot{\rho}_{e}^{i}=0.5 \dot{\rho}_{e}^{i, i+n} ; \quad \dot{\rho}_{e}^{i+n}=0.5 \dot{\rho}_{e}^{i, i+n}
$$

In general, the dislocation line direction $\vec{\xi}$ would not be aligned with the cell wall but is inclined with respect to it. In this case the generation will be the same, but a term $\left(\vec{n}_{w} \cdot \vec{\xi}\right) /\left(\left|\vec{n}_{w}\right||\vec{\xi}|\right)$ will be multiplied to the generation rate, where $\vec{n}_{w}$ is the cell wall normal. For example, the real generation rate on the positive edge of the $(i+1)^{t h}$ cell will be $\dot{\rho}_{e+}^{\text {wall }} \times\left(\vec{n}_{w} \cdot \vec{\xi}\right) /\left(\left|\vec{n}_{w}\right||\vec{\xi}|\right)$, meaning that even if the gradient is large between the $i^{t h}$ and $(i+1)^{t h}$ cells, if $\left(\vec{n}_{w} \cdot \vec{\xi}\right) /\left(\left|\vec{n}_{w}\right||\vec{\xi}|\right)=0$ (i.e. the dislocation line direction is perpendicular to the cell alignment), no generation will occur.

It is worth noting that the down scaling of the generation rates in eqn. (21) effectively represents an effect of the line tension to resist the dislocations from developing "curvatures", which, in the edge-screw idealization, are simply manifested by the lengthening of one dislocation character as the other character glides. This economical way of approximately treating curvature and line-tension effects avoids the heavy computation required in solving the coupled evolutions of density and curvature as in the CDD scheme (Hochrainer, Zaiser and Gumbsch 2007).

As mentioned in Section 2.3.1, cross slip for screw dislocations may be allowed in the present simulations. A cross-slip probability given as (Madec et al. 2002) $P_{\text {cross-slip }}=\mathrm{e}^{\left(-\frac{E_{A}}{k T}\right)}$ may be used, where $k$ is the Boltzmann constant, $T$ is the absolute temperature, and $E_{A}$ is the activation energy.

\subsection{Annihilation}

The annihilation process is shown in Fig. 6, where two groups of opposite signed dislocations, blue and red, situated in two neighboring cells at the time instant shown are approaching each other for annihilation. In the FVM implementation, while the computation is based on each cell, the annihilation process shown in Fig. 6 actually depends on dislocation densities of the opposite sign in the neighboring cells. Thus, using eqn. (17) for each cell, where $\rho_{e_{+}}, \rho_{e_{-}}, \rho_{s_{+}}$and $\rho_{s_{-}}$are evaluated only for the cell concerned, will not enable the annihilation process shown in Fig. 6 to be captured. To capture such a process, the dislocation densities in eqn. (17) need to be replaced by average values taken over neighboring cells. For this reason, a modified form of eqn. (17) is used in the actual implementation, namely, 


$$
\begin{aligned}
& \dot{\rho}_{e+}^{a n n}=\dot{\rho}_{e^{-}}^{a n n}=-\left[\min \left(\bar{\rho}_{e+}, \bar{\rho}_{e-}\right)\right]^{2} R_{e}\left|v_{e+}-v_{e-}\right| \\
& \dot{\rho}_{s+}^{a n n}=\dot{\rho}_{s-}^{a n n}=-\left[\min \left(\bar{\rho}_{s+}, \bar{\rho}_{s-}\right)\right]^{2} R_{s}\left|v_{s+}-v_{s-}\right|
\end{aligned}
$$

where

$$
\bar{\rho}_{e+}=\frac{1}{N_{a n n}} \sum_{R \leq R_{a n n}} \frac{\left(R_{a n n}-R\right) \rho_{e+}}{R_{a n n}} \text {, etc., }
$$

are weighted averages of the corresponding dislocation densities, taken over the $N_{\text {ann }}$ cells inside a radius of annihilation $R_{a n n}$, and $R$ is the distance between the current cell and the summation cell. With $R_{\text {ann }}$, which is much larger than the actual capture radius for annihilation $R_{e}$ or $R_{s}$, we can include some of the dislocation densities in neighboring cells in the evaluation of the opposite-signed densities for anticipated annihilation, so that cases like Fig. 6 can be captured. It should be noted that the use of $R_{\text {ann }}$ here is only for the evaluation of the average dislocation densities for anticipated annihilation, while the actual annihilation rates are still scaled by the capture radius for annihilation $R_{e}$ or $R_{s}$ which are of atomic dimensions.

\subsection{A Simple Simulation Example for Single Crystal Aluminum}

FORTRAN codes were written with the above implementation scheme. Here, we present a first example of numerical implementation involving a rectangular single crystal subjected to a uniaxial load $P$, as shown in Figure 7(a). Periodic boundary conditions were applied to all three dimensions, which means that as dislocations go out from one side of the simulation block, they re-enter at the opposite side. Initially, the density of each of the 48 types of dislocations was set to be randomly distributed within the simulation block, with a total value at $10^{12} \mathrm{~m}^{-2}$. The cell size $(\Delta x, \Delta y, \Delta z)$ used in the FVM scheme was $(200 b \times 200 b \times 200 b)$, and the simulation block had $50(x) \times 50(y) \times 5(z)$ cells (Table 1$)$. The time step in the simulation was adjusted using a simple adaptive scheme of

$$
\Delta t=0.5 \times(\Delta x \Delta y \Delta z)^{\frac{1}{3}} / \max (\text { speed of the densities })
$$

In this exercise, we aim to replicate the crystal plasticity response of pure aluminum. Thus, the following material parameters for $\mathrm{Al}$ were used in the simulations: $b=2.863 \times 10^{-10} \mathrm{~m}$ and $\mu=25 \times 10^{9} \mathrm{~Pa}$. The parameters in dislocation velocity law in eqn. (2) were chosen to represent the mean behavior between screw and edge dislocations as calculated from molecular dynamics simulation by Olmsted et al. (2005): $\quad v_{0}=1 \mathrm{~m} / \mathrm{s}, \quad \tau_{0}=0.0463 \mathrm{MPa}$, and $m=1$. The out-of-plane Taylor hardening coefficient in eqn. (13) was set at $\alpha_{\perp}=0.3$, while the in-plane hardening 
coefficient in eqn. (14) was at $a_{\|}=0.1$.

The tensile stress was applied along the $\langle 100\rangle,\langle 111\rangle,\langle 112\rangle$ or $\langle 123\rangle$ directions of the fcc lattice, and the strain rate was set to be $2 \times 10^{-3} \mathrm{~s}^{-1}$. Figure 7(b) shows the simulated stress-strain responses, in comparison with the experimental data by Hosford et al. (1960). Overall, the simulations correctly predicted the experimental trend that the $\langle 111\rangle$ orientation is strongest, while the $\langle 112\rangle$ and $\langle 123\rangle$ orientations are weakest. For the $\langle 111\rangle$ orientation, the initial strain hardening, up to about $0.5 \%$, is rather well captured by the simulation, although at higher strains, the simulated hardening is not as rapid as the experimental value. For the other three orientations $\langle 100\rangle,\langle 112\rangle$ and $\langle 123\rangle$, the simulation overestimates the initial flow stress, but better agreement is achieved for larger strains. Several factors may account for the discrepancies from the experimental data. First, the Taylor interactions may not be fully represented by the reduced scheme in eqns. (13) and (14). Secondly, the accumulation of dislocations may not be fully represented by the scheme in eqns. (20) and (21) - such a scheme accounts only for production due to the connectivity of dislocations, namely, new dislocations are produced whenever gradients in flux arise (Figure 3), but generation from discrete sources is not modeled. In any case, Figure 7(b) shows that the flow anisotropy in $\mathrm{Al}$ is captured by the simulations.

The case with the tensile direction along $\langle 100\rangle$ is selected for more detailed analysis in Figure 8. From Figure 8(a), the normal strain component $\varepsilon_{x x}$ along the tensile axis increases in the tensile direction with time, while $\varepsilon_{y y}$ and $\varepsilon_{z z}$ along the orthogonal directions also develop with time according to $\varepsilon_{x x}+\varepsilon_{y y}+\varepsilon_{z z} \approx 0$, as must be the case since volume is conserved. Figure $8(\mathrm{~b})$ shows the simulated stress-strain behavior. From Figure 8(c), the dislocation densities on the four sets of slip planes multiply steadily, to the order of $10^{14} \mathrm{~m}^{-2}$ at the strain of about $5 \%$. Figure $8(\mathrm{~d})$ shows the evolution of the Taylor hardening resistance and the dislocation elastic interaction stress. The contribution from GNDs in Taylor hardening is also calculated by counting only the dislocation contents with a net Burgers vector in each simulation cell, and plotted separately in Figure 8(d). It can be seen that, although GNDs play a major role in Taylor resistance, the hardening due to the SSDs, i.e. the discrepancy between total hardening stress and GND hardening, is certainly not negligible. The Taylor hardening and elastic interaction both increase as the dislocation density increases. The elastic interaction is negligibly small at the beginning of the simulation, due to the nearly even distribution of dislocations. However, as the simulation proceeds, the elastic interaction between dislocations becomes more significant.

Of interest here is that instead of a smooth behavior, stress bumps are seen throughout the plastic flow process in Figure 8(b). Such a bumpy flow behavior is not 
expected from a bulk specimen, but it must be remembered that the current stress-strain behavior in Figure 8(b) is computed for a small simulation block size of $50(x) \times 50(y) \times 5(z)$ cells, or $2.86 \mu \mathrm{m} \times 2.86 \mu \mathrm{m} \times 0.286 \mu \mathrm{m}$. For small crystals of such a size, stress bumps are often seen in real experiments due to a lack of mean-field environment for dislocation interactions (Rao et al. (2008); Ng and Ngan (2008)). In a bulk specimen, discrete events in different parts of the specimen would average out to give a smooth overall response. The stress-strain response in Figure 8(b) is therefore reasonable.

Figure 8(e-h) illustrates the evolution of dislocation density patterns from the simulation in Figure 8(b). At the beginning, the dislocations are very evenly distributed with a low density. As strain increases, the dislocation density in some regions builds up rapidly, while that in other regions stagnates or even declines. Regions with either very high dislocation densities reaching $10^{14} \mathrm{~m}^{-2}$, or very low dislocation densities at $10^{12} \mathrm{~m}^{-2}$, appear. The areas with high and low densities juxtapose and form alternating patterns resembling a cellular structure.

\section{A Detailed Case Study: Size Effect of Crystal Plasticity}

\subsection{Motivation}

To further validate the present DDFD approach, we have performed a detailed case study on the size dependence of strength of small crystals. It is by now well-known that nano- or micron- sized crystals in general deform in a jerky manner with stochastic occurrence of discrete strain bursts (Uchic et al. 2004, Ng and Ngan 2008, Shan et al. 2008, Kim et al. 2012). In addition, their yield strength $\sigma$ exhibits a remarkable smaller-being-stronger size effect according to a power law $\sigma \sim D^{-m}$, where $D$ is specimen size (Greer and De Hosson 2011; Dou and Derby 2009). For sub-micron sized specimens, the reason for such a size effect and jerky deformation is easy dislocation depletion from the specimen volume and the need for re-nucleation (Greer and Nix 2006), and this so-called dislocation starvation mechanism has been confirmed by in situ TEM experiments (Shan et al. 2008). For larger specimens in the micron regime, complete starvation is difficult to achieve, and the size effect has been proposed to be a consequence of the size dependence of the dislocation microstructure the specimen contains - smaller specimens were proposed to either contain shorter dislocation source lengths (Parthasarathy et al. 2007, Akarapu 2010), or finer dislocation mesh size (Gu and Ngan 2013).

DDD simulations have been employed to understand small-scale plasticity, but due to the amount of computation involved in 3D DDD, previous attempts were limited to the small specimen size range of sub-micron to a few microns, with 
dislocation densities of $10^{12}$ to $10^{15} \mathrm{~m}^{-2}$ (Akarapu et al. 2010, Zhou et al. 2010, Huang et al. 2012) and much higher strain rates, on the order of $10^{2} \mathrm{~s}^{-1}$ or above, compared to experimental conditions (Motz et al. 2009, Akarapu et al. 2010, Zhou et al. 2010). A so-called 2.5D DDD approach (Benzerga 2009, Gómez-García et al. 2006) has also been used, which is a mesoscopic simulation scheme based on 2D DDD but with certain 3D mechanisms, such as Taylor interactions and Frank-Read sources, partially considered in a highly simplified manner. MD simulations have also been carried out (Yamakov et al. 2002, Komanduri et al. 2001, Xu et al. 2013), but as mentioned beforehand, real time and size scales were not simulated directly. For these reasons, there is a need for an approach which can capture small-scale plasticity over a wide range of specimen size from, say, a micron to tens or hundreds of microns, in a unified way. The dislocation-density based CDD scheme has been employed to model size effects of thin films under tension and bending (Zaiser et al. 2007; Sandfeld et al. 2010; Sandfeld et al. 2011). However, because of the very heavy computational costs involved, simple geometries involving only two slip systems in a symmetrical configuration were simulated, and no comparison with experimental results was made. In the following, the use of the present DDFD scheme to simulate small-scale plasticity in an fcc model with 12 slip systems of the type $\{111\}\left\langle\frac{1}{2}, \frac{1}{2}, 0\right\rangle$ is described.

\subsection{Simulation Details}

Single fcc crystals of various sizes were constructed in the $\left[\begin{array}{lll}1 & 0 & 0 \\ 0 & 1 & 0 \\ 0 & 0 & 1\end{array}\right]$ orientation, with the tensile load applied along [001], i.e. the $z$-direction. The surfaces normal to the $x$ - and $y$ - axes were set to be free surfaces, while periodic boundary conditions were applied along the z-axis, so that the specimen can be thought of as a wire of infinite length along the z-axis. The pixel size was $(100 \mathrm{~b}, 100 \mathrm{~b}$, $100 b)$ for the smallest specimen of size $(1000 b, 1000 b, 1000 b)$, and was $(200 b, 200 b$, $200 b)$ for other specimen sizes of $(2000 b, 2000 b, 1000 b)$, $(4000 b, 4000 b, 1000 b$,$) ,$ $(6000 b, 6000 b, 1000 b), \quad(8000 b, 8000 b, 1000 b)$ and $(16000 b, 16000 b, 1000 b)$. The simulation scheme followed the same way as described in Section 3. In the simulation, the dislocation speed was capped at $20 \mu \mathrm{m} / \mathrm{s}$, and the prototypic values of Taylor interaction coefficients employed were $\alpha_{\perp}=1$ and $a_{\|}=0.5$ (c.f. section 2.2.2). Activation energy for cross-slip was assumed to be $0.5 \mathrm{eV}$ (c.f. section 3.3), and the stress exponent $m$ in the velocity law in eqn. (2) was set to be 1. Other material parameters were: $b=2.863 \times 10^{-10} \mathrm{~m}, \mu=26 \times 10^{9} \mathrm{~Pa}, \tau_{0}=7.8 \times 10^{6} \mathrm{~Pa}$ and $v_{0}=1 \mathrm{~ms}^{-1}$. The initial density of each of the 48 dislocation types in each 
simulation pixel was generated randomly from a Gaussian distribution with mean $\frac{10^{12} \mathrm{~m}^{-2}}{48}$ and standard deviation $10^{9} \mathrm{~m}^{-2}$, which is simply a convenient choice. This corresponds to an overall averaged dislocation density of $10^{12} \mathrm{~m}^{-2}$.

The crystals were loaded at a constant nominal strain rate of $2.5 \times 10^{-3} \mathrm{~s}^{-1}$ which is a representative experimental value. One feedback method used is to adjust the stress in proportion to the error (El-Awady et al. 2009), i.e.

$$
\dot{\boldsymbol{\sigma}} \propto\left(\dot{\varepsilon}_{\text {prescribed }}-\dot{\varepsilon}_{\text {actual }}\right)
$$

While this works pretty well in most cases, for smaller specimens where extensive dislocation depletion frequently occurs, serious overshoot in stress may occur whenever dislocation depletion and a tremendous drop in strain rate happen, so that the applied stress is increased rapidly. To alleviate such a problem, a derivative term similar to that used in proportional-integral-differential control was added to the stress adjustment, i.e. the stress was adjusted also in proportion to the rate of change of the strain-rate error. If the error in strain rate is decreasing, the stress adjustment is also decreased and vice versa. This is found to be effective in suppressing the stress overshooting.

In addition to the externally applied stress, image stresses which pull dislocations towards free surfaces are normally considered in discrete DD simulations (Fivel and Canova 1999). While it is not particularly difficult to include image stresses in the present work, this is not done because the pixel size in the present approach is typically hundreds of Burgers vectors large. Since image stresses attenuate from the free surface over distances that scale with $b$, the image stress acting even in the first pixel next to a free surface would be negligible. For this reason, image stresses are ignored in the present work. All the free surfaces of the simulation block adopt a no-entry, free-exit boundary condition, i.e. no dislocation flux enters the simulation space through the free surfaces, but dislocation fluxes are not obstructed from leaving the simulation space at the free surfaces. Such a condition constantly leaves the simulation cells at the free surfaces at a lower dislocation density than the interior cells.

The no-entry, free-exit boundary condition is coupled with the MUSCL scheme by using ghost cells with velocity and density values assigned as follows:

For $i=1$ or $j=1$ or $k=1$ : 


$$
\left\{\begin{array}{c}
v_{i-1}=\min \left(0, v_{i}\right), \\
\rho_{i-1}=\rho_{i} \max \left(0, \operatorname{sgn}\left(-v_{i}\right)\right), \quad \text { and } \\
(\rho v)^{*}{ }_{i-1 / 2}=\min \left(0,(\rho v)^{*}{ }_{i-\frac{1}{2}}\right)
\end{array}\right.
$$

For $i=n x$ or $j=n y$ or $k=n z$ :

$$
\left\{\begin{array}{c}
v_{i+1}=\max \left(0, v_{i}\right), \\
\rho_{i+1}=\rho_{i} \max \left(0, \operatorname{sgn}\left(v_{i}\right)\right), \text { and } \\
(\rho v)^{*}{ }_{i+1 / 2}=\max \left(0,(\rho v)^{*}{ }_{i+\frac{1}{2}}\right)
\end{array}\right.
$$

where $v$ and $\rho$ are the velocity and density of type $\alpha$ dislocations. When $v$ inside the free surface cell is pointing outward of the simulation space, the ghost cell would be at a downwind position, and the velocity and density of type $\alpha$ dislocations in the ghost cell would be equal to that of the surface cell, so that the flux out of the simulation space would not be obstructed. This is referred to as the zero-streamwise gradient exit condition (Zikanov 2010). On the other hand, if the velocity of type $\alpha$ dislocations in the surface cell points inward of the simulation space, the ghost cell would be upwind of the surface cell. Since there should be zero flux entering the simulation space, the ghost cell would be assigned zero dislocation density and zero velocity.

\subsection{Simulation Results}

Figure 9 shows the simulated stress-strain curves of specimens of various sizes. It can be seen that the tensile strength increases with decreasing specimen size, and the smaller specimens exhibit a sharp yield point. The upper yield stress of the first yield point represents the strength of the specimens, and this is plotted in Figure 10 versus specimen size in a double-logarithmic fashion. The variation between the present simulated strength $\sigma$ and size $D$ roughly follows the well-known power-law trend $\sigma \sim D^{-m}$. Also shown in Figure 10 are typical experimental data and calculated yield stresses, and it can be seen that the present results are in broad agreement with known reported data.

Of special interest in the results so far is that a sharp initial yield point occurs for specimens $<4000 b$ in Figure 9, and this diminishes for larger specimens. Figure 11 shows the total dislocation density evolution during deformation. It can be seen that the smaller specimens first undergo a considerable drop in dislocation density, and eventually end up with an almost constant dislocation density, whereas the larger specimen simulated of size of $16000 \mathrm{~b}$, i.e. $\sim 4.6 \mu \mathrm{m}$, first undergoes an insignificant change in the dislocation density initially, followed by a subsequent gradual build-up. 
By noting that strain is proportional to time at constant strain-rate, the stress peaks in the stress-strain curves for the smaller specimens in Figure 9 are found to correspond to the initial depletion and then sudden multiplication of dislocation density in Figure 11 , and so their formation is related to the loss of dislocations from the specimen, i.e. dislocation starvation. The stress peak therefore has the same nature as a strain burst accompanying mechanical annealing (Shan et al. 2008): with a small specimen cross-section, the initial dislocations present quickly escape from the specimen at the initial stage of plastic deformation, and once the specimen becomes depleted of dislocations, the deformation cannot catch up with the prescribed strain rate. The applied stress then increases rapidly to a high value at which rapid dislocation multiplication occurs. The instantaneous strain rate then momentarily exceeds the prescribed strain rate, and so the applied stress decreases rapidly to maintain the strain rate. The initial stress overshoot is therefore attributed to the low initial dislocation density level with respect to the prescribed constant strain rate (Yonenaga and Sumino 1978; Verdier et al. 1998; Petukhov 2001). Whenever the prescribed strain rate is high and the initial dislocation density small, stress has to increase rapid to a level for dislocation multiplication to occur. When enough dislocations have been generated, the strain rate would exceed the prescribed value, and the stress would start to relax. The largest specimen size simulated here is $16000 \mathrm{~b}$ which corresponds to only several microns, and the stress overshoot is known to occur in this size regime. In the classical tensile experiments on metal whiskers by Brenner (1957), the whiskers used were a few to over a dozen microns in diameter, and high ratios of yield stress to flow stress of up to $\sim 80: 1$ were observed. The whiskers Brenner used were not pre-strained, and so their initial dislocation density should also be low.

Figure 12 shows the 3-D dislocation-density plots for different specimen sizes at different cumulative strains. As in Figure 11, for the smaller specimen sizes $1000 b$ and $4000 \mathrm{~b}$, the dislocation density remains at a low value, but for the largest specimen size of $16000 \mathrm{~b}$ simulated, dislocation accumulation takes place as strain increases. At a plastic strain of $\sim 0.2 \%$, clear dislocation debris is observed on $\{111\}$ planes (Ohashi 2005). Also, while the dislocations are concentrated in the core region for the smaller specimens, for larger specimens, clusters of dislocations are scattered around the whole specimen, with a relatively lower density at the free surfaces. The clustered cases correspond well to the so-called "soft-surface, hard-core" structure commonly observed in experiments (Fourie 1970), in which the observed dislocation density is higher in the core region. In addition, the low dislocation density in the proximity of free surfaces indicates that free surfaces are effective sinks for dislocations. Thus, in the initial stage of deformation where the dislocation generation rate is low, the rate of dislocation escape is the highest in the smallest specimen due to the highest surface 
area to volume ratio.

From the simulation results, the reason for present model to capture the size effect of strength in Figure 10 is summarized as follows. The free surfaces normal to the $\mathrm{x}$ - and $\mathrm{y}$ - axes of the specimens allow dislocations to exit the simulation space freely (c.f. eqns. (26) and (27)), and hence they are effective sinks for dislocations. Since a smaller specimen has a higher surface area to volume ratio, the loss rate of dislocations at free surfaces would increase with decreasing specimen size. Figure 11 shows that the largest specimen has its dislocation density increasing with strain, while for the smaller specimens, the dislocation density initially rises and then settles to a steady state. By analyzing the simulation data, such a steady state was found to be an equilibrium condition in which the loss rate of dislocations at free surfaces is approximately balanced by the dislocation generation rate. Since the loss rate at free surfaces is higher in a smaller specimen, and the generation rate at steady state is also higher for a smaller specimen. Since the generation rate scales with the work done by stress (eqn. (21)), a higher generation rate would require a higher flow stress, so a smaller specimen is predicted to be stronger.

In summary, the use of the DDFD scheme here successfully captures a number of key features of small-scale plasticity. First, jerky deformation typically seen in experiments (Uchic et al. 2004, Ng and Ngan 2008, Shan et al. 2008, Kim et al. 2012) is reproduced in Figure 9. Secondly, the "smaller-being-stronger" size effect is reproduced in Figure 9, and strength was found to roughly follow a power-law trend with size, in agreement with known experimental data as shown in Figure 10. The storage of dislocations as seen from Figure 11 and 12 is also in agreement with experimental findings - for small specimens, storage is low with easy depletion from the specimen (Shan et al. 2008; Ng and Ngan 2008), and for large specimens where storage is efficient, the experimental "soft-surface, hard-core" structure (Fourie 1970) is reproduced. The broad agreement between the computational results and experimental observations here indicates the validity of the present DDFD approach. As discussed above, the prime factor for the "smaller-being-stronger" size effect is the easy loss of dislocations from small specimens, which is a direct consequence of the continuity nature of dislocation movements. The current DDFD approach takes the continuity nature of dislocation movements explicitly into consideration via eqn. (3), and this is an important reason why size effect can be captured as shown in Figure 10.

\section{Discussion}

\subsection{Significance of Elastic Interactions and SSDs}

As explained in the Introduction, the key differences between the present model 
and earlier ones are (i) a formal treatment of the mutual elastic interactions between dislocations via the Hirth-Lothe formulation, and (ii) the employment of the same dynamics laws for both SSDs and GNDs, instead of treating them separately as in a crystal kinematics framework. Here, it is useful to see the significance of both features in the numerical examples presented above.

In the simulation for $\mathrm{Al}$ with periodic boundary conditions in Section 3.5, Figure 8(d) shows that Taylor hardening is the major component of strength, in agreement with the experimental observation that the strength of micro-specimens is well correlated to the square-root of the dislocation density stored inside them $(\mathrm{Gu}$ and Ngan 2012). However, Figure 8(d) still shows that the elastic interactions between dislocations are about $10 \%$ of the Taylor resistance and hence are not negligible. In the case study on small specimens with free surfaces discussed in Section 4, the elastic interactions between dislocations produce more intricate effects. Figure 13 shows the effects of switching off the elastic interactions between dislocations in this case. Comparing Figure 13(a) with Figure 11 shows that with elastic interactions switched off, all specimens, including the largest simulated of $16000 \mathrm{~b}$, do not exhibit growth in dislocation density on continuous deformation, although growth is still expected in larger specimen sizes not simulated. Comparing Figure 13(b) with Figure 12 shows that switching off elastic interactions suppresses the occurrence of high dislocation densities - the maximum densities encountered in Figure 12 well exceed $4 \times 10^{12} \mathrm{~m}^{-2}$, while those in Figure 13(b) are significantly lower, and this also agrees well with the lack of growth in Figure 13(a). The $0.2 \%$ proof stress data in Table 2 indicate that switching off the elastic interactions leads to significant overestimation of the proof stress for the smallest specimen simulated, and this trend drops on increasing specimen size, ending with a slight underestimation of the strength for the largest specimen simulated. Analyzing the dislocation loss rate at free surfaces and generation rate in the specimen interior shows that both rates increase without elastic interactions, yet they again more or less balance one another corresponding to the steady density in Figure 13(a). The results here therefore indicate that switching off the elastic interactions reduces high-density dislocation entanglements, so that dislocations can exit a small specimen through its free surfaces more easily. To maintain the prescribed strain rate, the generation rate within the specimen has to increase, and this requires higher stress, thus explaining the higher strength for most specimen sizes in Table 2 when elastic interactions are switched off. This trend, however, is counteracted by the reduced Taylor hardening as high-density dislocation clusters are suppressed with elastic interactions turned off, and this explains why the largest specimen becomes softer without such interactions. In summary, the present results show that mutual elastic interactions between dislocations produce intricate yet 
significant effects on the dislocation microstructure and flow resistance especially in small specimens.

On the effects of SSDs in the overall flow resistance, in the example in Figure 8(d), the net effects of SSDs on the Taylor hardening are revealed as the difference between the total hardening and that due to the GNDs. In this example, SSDs account for about $10 \%$ of the Taylor resistance, and this again is not negligible.

\subsection{Elastic Deformation and Lattice Rotations}

The DDFD formulation described in sections 2 and 3 focusses on the plastic strain given as the time-integration of the Orowan strain rate, viz. eqns. (4) and (5), while elastic deformation has been neglected so far. It is useful here to understand the significance of such a plastic strain in a general elastoplastic framework. In the crystal kinematics approach of plasticity (Asaro and Rice 1977), the total deformation gradient tensor $\boldsymbol{F}$ is decomposed into a plastic component $\boldsymbol{F}^{\boldsymbol{p}}$ as well as an elastic component $\boldsymbol{F}^{\boldsymbol{e}}$, i.e. $\boldsymbol{F}=\boldsymbol{F}^{\boldsymbol{e}} \boldsymbol{F}^{\boldsymbol{p}}$, and $\boldsymbol{F}^{\boldsymbol{p}}$ and $\boldsymbol{F}^{\boldsymbol{e}}$ are related to the plastic and elastic deformation tensors $\boldsymbol{U}^{\boldsymbol{p}}$ and $\boldsymbol{U}^{\boldsymbol{e}}$ as $\boldsymbol{F}^{\boldsymbol{p}}=\boldsymbol{I}+\boldsymbol{U}^{\boldsymbol{p}}$ and $\boldsymbol{F}^{\boldsymbol{e}}=\boldsymbol{I}+\boldsymbol{U}^{\boldsymbol{e}}$ respectively. The plastic strain $\varepsilon^{p}(\vec{r}, t)$ calculated via eqns. (4) and (5) represents a coarse-grained, pure-shear strain tensor $\boldsymbol{U}_{\|}^{p}$ produced by the glide motion of the GNDs, while their self-fields are not represented (Figure 14). The discreteness of the slip steps produced after the glide motion is also not included in $\boldsymbol{U}_{\|}^{p}$, but this is unimportant at the coarse-grained resolution concerned. Also, by virtue of the summation in eqn. (4), the glide motions of the SSDs will cancel out at the coarse-grained resolution concerned, but, as mentioned in section 2.2.1, the dynamics of the GNDs are intricately affected by those of the SSDs and these are duly considered in the present model. The remaining part of the plastic deformation, $\boldsymbol{U}_{\perp}^{p}$ in Figure 14, is due to the self-fields of the GNDs, since strains of the SSDs will cancel out at the resolution of the present DDFD scheme concerned. More precisely, $\boldsymbol{U}_{\|}^{\boldsymbol{p}}$ is the anti-symmetric part of $\boldsymbol{U}^{\boldsymbol{p}}$ that switches sign as the dislocation contents reverse their motion (Figure 14), and $\boldsymbol{U}_{\perp}^{p}$ is the remaining symmetric part of $\boldsymbol{U}^{p}$, and $\boldsymbol{U}^{\boldsymbol{p}}=\boldsymbol{U}_{\|}^{p}+\boldsymbol{U}_{\perp}^{p}$. Similarly, if we define $\boldsymbol{U}_{\perp}^{\boldsymbol{e}}$ as the part of the elastic deformation due to the self-fields of the GNDs, and $\boldsymbol{U}_{\|}^{\boldsymbol{e}}$ as the remaining part of the elastic deformation due to the applied loadings, then $\boldsymbol{U}^{\boldsymbol{e}}=\boldsymbol{U}_{\perp}^{\boldsymbol{e}}+\boldsymbol{U}_{\|}^{\boldsymbol{e}}$. Note that $\boldsymbol{U}_{\|}^{\boldsymbol{p}}$ and $\boldsymbol{U}_{\|}^{\boldsymbol{e}}$ are compatible deformation tensors $\left(\boldsymbol{U}_{\|}^{\boldsymbol{p}}\right.$ is in fact pure shear), while $\boldsymbol{U}_{\perp}^{p}$ and $\boldsymbol{U}_{\perp}^{\boldsymbol{e}}$ contain incompatible components due to the GND contents present. In fact, the Nye tensor is given as $\boldsymbol{A}=-\operatorname{curl} \boldsymbol{U}_{\perp}^{\boldsymbol{p}}=\operatorname{curl} \boldsymbol{U}_{\perp}^{\boldsymbol{e}}$ (Taupin et al. 2008) and is non-zero in general, while curl $\boldsymbol{U}_{\|}^{\boldsymbol{p}}=\operatorname{curl} \boldsymbol{U}_{\|}^{\boldsymbol{e}}=\mathbf{0}$ due to the compatibility of $\boldsymbol{U}_{\|}^{p}$ and $\boldsymbol{U}_{\|}^{e}$. The total deformation mapping is therefore $\boldsymbol{F}=\boldsymbol{F}^{\boldsymbol{e}} \boldsymbol{F}^{\boldsymbol{p}}=\left(\boldsymbol{I}+\boldsymbol{U}_{\perp}^{\boldsymbol{e}}+\boldsymbol{U}_{\|}^{\boldsymbol{e}}\right)\left(\boldsymbol{I}+\boldsymbol{U}_{\perp}^{\boldsymbol{p}}+\boldsymbol{U}_{\|}^{\boldsymbol{p}}\right)$, and this can be expressed as 


$$
F=I+U_{\text {overall }}+U_{\text {internal }}
$$

where

$$
U_{\text {overall }}=U_{\|}^{p}+U_{\|}^{e}\left(I+U_{\|}^{p}\right)
$$

may be described as the "overall" elasto-plastic deformation due to the motion of the GNDs, without considering their self-fields. Also, in eqn. (28),

$$
U_{\text {internal }}=\left(U_{\perp}^{p}+U_{\perp}^{e}\right)+U_{\|}^{e} U_{\perp}^{p}+U_{\perp}^{e} U^{p}
$$

is the "internal" deformation due to the self-fields of the GNDs.

The above indicates that the present DDFD scheme offers a method whereby the plastic part $\boldsymbol{U}_{\|}^{\boldsymbol{p}}$ of the $\boldsymbol{U}_{\text {overall }}$ deformation in eqn. (29) can be calculated. Although not done in the examples in this work, the remaining elastic part $\boldsymbol{U}_{\|}^{\boldsymbol{e}}\left(\boldsymbol{I}+\boldsymbol{U}_{\|}^{\boldsymbol{p}}\right)$ can also be calculated rather easily, as a purely elasticity exercise using the plastically deformed shape $\left(\boldsymbol{I}+\boldsymbol{U}_{\|}^{\boldsymbol{p}}\right)$ as a reference state subjected to the external loading, without considering the dislocations inside.

For $\boldsymbol{U}_{\text {internal }}$, eqn. (30) indicates that it is first order in $\left(\boldsymbol{U}_{\perp}^{\boldsymbol{p}}+\boldsymbol{U}_{\perp}^{\boldsymbol{e}}\right)$, which is simply the elasto-plastic deformation due to the self-fields of the GND contents. This term gives important information about local lattice rotations and strain gradients, and although their full solution would be a very involved exercise, the key information can in fact be represented by the Nye tensor which, in the present simulation scheme, can be easily calculated as $\boldsymbol{A}=\sum_{\alpha}\left(\rho_{\alpha}^{+}-\rho_{\alpha}^{-}\right) \vec{b}_{\alpha} \otimes \vec{\xi}_{\alpha}$ (Arsenlis et al. 2004). The case study in Section 4 above concerns uniform deformation of small crystals where lattice rotations and local strain gradients are not as important as the loss of dislocations which gives rise to the size effect of strength. However, in other cases, lattice rotations, which are pertinent to dislocation pattern formation, may be of significant interest and should not be ignored. For example, the Nye tensor is very useful in indicating subcell formation under vibrational loadings and this will be discussed elsewhere (Cheng et al., 2014). Also, as future work, the lattice rotations associated with the computed Nye tensor can be used to update the resolved shear stress on the slip systems at each time step, in order to provide a strategy to more accurately capture the larger-strain range.

\section{Conclusions}

A dislocation-density functional model for crystal plasticity has been developed which takes into full consideration of the mutual elastic interactions between 
dislocations, as well as the following elements of the dislocation theory: (i) the continuity nature of dislocation movements, (ii) forest hardening, (iii) generation according to high spatial gradients in dislocation densities, and (iv) annihilation. Equations governing the evolution of dislocation density functions have been derived based on consideration of dislocation fluxes with the above elements incorporated. Numerical implementation has been designed with the finite-volume method, which is well suited for flux problems with high gradients, as in the dislocation case. Numerical examples have been performed for an idealized single-crystal aluminum model with 48 types of dislocation densities on 12 slip systems. Typical strength anisotropy behavior comparable to experimental observations was predicted. A detailed case study on small-scale crystal plasticity also successfully captured a number of key experimental features, including power-law relation between strength and size, low dislocation storage and jerky deformation. The size effect of strength, which originates from the easy loss of dislocations from a small material volume, illustrates the importance of the continuity nature of dislocation movements duly considered in the present simulation approach.

\section{Appendix}

The interactive glide stress $\tau_{\alpha}^{\text {int }}$ is given by eqn. (10) involving a kernel $g(\vec{R}, t)$ given in eqn. (11). Although not used in this work, the climb component of the interactive stress can also be obtained from the last term in eqn. (8) as $\tau_{\alpha}^{\text {climb }}=\vec{f}_{\alpha}$. $(\hat{\xi} \times \hat{b}) / b$, giving

where

$$
\tau_{\alpha}^{c l i m b}(\vec{r}, t)=\sum_{\alpha \prime} \iiint h(\vec{R}, t) \rho_{\alpha^{\prime}}\left(\vec{r}^{\prime}, t\right) d^{3} r^{\prime}
$$

$$
\begin{aligned}
h(\vec{R}, t)= & -\frac{\mu}{8 \pi}(\hat{\xi} \times \hat{b}) \cdot\left[\left(\vec{b}^{\prime} \times \hat{b}\right) \cdot \vec{\nabla}\left(\nabla^{2} R\right)\right]\left(\hat{\xi} \times \hat{\xi}^{\prime}\right) \\
& -\frac{\mu}{8 \pi}(\hat{\xi} \times \hat{b}) \cdot\left\{\left[\vec{b}^{\prime} \times \vec{\nabla}\left(\nabla^{2} R\right)\right] \times \hat{\xi}\right\}\left(\hat{b} \cdot \hat{\xi}^{\prime}\right) \\
& -\frac{\mu}{4 \pi(1-v)}(\hat{\xi} \times \hat{b}) \cdot\left[\left(\vec{b}^{\prime} \times \hat{\xi}^{\prime}\right) \cdot \vec{\nabla}\right](\hat{\xi} \times \hat{b} \mathbb{T}) \\
& +\frac{\mu}{4 \pi(1-v)}\left[\left(\vec{b}^{\prime} \times \hat{\xi}^{\prime}\right) \cdot \vec{\nabla}\left(\nabla^{2} \mathrm{R}\right)\right] .
\end{aligned}
$$

Here, we evaluate the kernels $g(\vec{R})$ and $h(\vec{R})$.

\section{Case (i) - the two dislocation densities are non-parallel}

In Figure 1(a), the oblique coordinates $x$ and $y$ are the distances from $O$ to 
the segments $d-1$ and $d-2$ along the dislocation line vectors $\hat{\xi}_{1}$ and $\hat{\xi}_{2}$ respectively, and $z$ is along direction $\hat{e}_{3}$ given by

$$
\hat{e}_{3}=\frac{1}{\sin \theta}\left(\hat{\xi}_{1} \times \hat{\xi}_{2}\right)
$$

where $\theta$ is the angle between $\hat{\xi}_{1}$ and $\hat{\xi}_{2}$. The distance $R$ between the two segments is then given by $R=\left(x^{2}+y^{2}-2 x y \cos \theta+z^{2}\right)^{1 / 2}$, and the gradient operator $\vec{\nabla}=\hat{R}(d / d R)$ in terms of the oblique coordinates is (Hirth and Lothe 1992)

$$
\vec{\nabla}=\hat{\xi}_{1}\left(\frac{1}{\sin ^{2} \theta} \frac{\partial}{\partial x}+\frac{\cos \theta}{\sin ^{2} \theta} \frac{\partial}{\partial y}\right)-\hat{\xi}_{2}\left(\frac{1}{\sin ^{2} \theta} \frac{\partial}{\partial y}+\frac{\cos \theta}{\sin ^{2} \theta} \frac{\partial}{\partial x}\right)+\hat{e}_{3}\left(\frac{\partial}{\partial z}\right)
$$

In Figure 1(a), the relative positions between two interacting short dislocation segments $d-1$ and $d-2$ are specified by $(x, y, z)$ in the oblique coordinate system. Let the relative position of $d-1$ from $d-2$ be $\vec{R}=\left(x_{1}, x_{2}, x_{3}\right)$ in a global Cartesian coordinate system. The global coordinates $\left(x_{1}, x_{2}, x_{3}\right)$ of $\vec{R}$ are related to the $(x, y, z)$ in the oblique coordinate system via the transformation

$$
\left[\begin{array}{l}
x \\
y \\
z
\end{array}\right]=\left[-\left[\begin{array}{lll}
\xi_{1} & {\left[\xi_{2}\right.}
\end{array}\right] \quad\left[e_{3}\right]\right]^{-1}\left[\begin{array}{l}
x_{1} \\
x_{2} \\
x_{3}
\end{array}\right]
$$

where $\left[\xi_{1}\right],\left[\xi_{2}\right]$ and $\left[e_{3}\right]$ are column vectors of $\hat{\xi}_{1}, \hat{\xi}_{2}$ and $\hat{e}_{3}$ respectively.

A particular simple result pertinent to eqns. (11) and (A2) is $\nabla^{2} R=2 / R$. The third term in eqns. (11) and (A2) involves the tensor $\mathbb{T}$, which is given in terms of the basis vectors in the oblique coordinate system (Hirth and Lothe 1992) as:

$$
\begin{aligned}
\mathbb{T}= & \hat{\xi}_{2} \otimes \hat{\xi}_{2}\left(\frac{\cos ^{2} \theta}{\sin ^{4} \theta} \frac{\partial^{2} R}{\partial x^{2}}+2 \frac{\cos \theta}{\sin ^{4} \theta} \frac{\partial^{2} R}{\partial x \partial y}+\frac{1}{\sin ^{4} \theta} \frac{\partial^{2} R}{\partial y^{2}}\right) \\
& -\hat{\xi}_{1} \otimes \hat{\xi}_{2}\left(\frac{\cos \theta}{\sin ^{4} \theta} \frac{\partial^{2} R}{\partial x^{2}}+\frac{1+\cos ^{2} \theta}{\sin ^{4} \theta} \frac{\partial^{2} R}{\partial x \partial y}+\frac{\cos \theta}{\sin ^{4} \theta} \frac{\partial^{2} R}{\partial y^{2}}\right) \\
& -\left(\hat{e}_{3} \otimes \hat{\xi}_{2}+\hat{\xi}_{2} \otimes \hat{e}_{3}\right)\left(\frac{\cos \theta}{\sin ^{2} \theta} \frac{\partial^{2} R}{\partial x \partial z}+\frac{1}{\sin ^{2} \theta} \frac{\partial^{2} R}{\partial y \partial z}\right) \\
& +\hat{\xi}_{1} \otimes \hat{e}_{3}\left(\frac{1}{\sin ^{2} \theta} \frac{\partial^{2} R}{\partial x \partial z}+\frac{\cos \theta}{\sin ^{2} \theta} \frac{\partial^{2} R}{\partial y \partial z}\right)+\hat{e}_{3} \otimes \hat{e}_{3} \frac{\partial^{2} R}{\partial z^{2}}
\end{aligned}
$$

where terms of $\hat{\xi}_{1} \otimes \hat{\xi}_{1}, \hat{\xi}_{2} \otimes \hat{\xi}_{1}$ and $\hat{e}_{3} \otimes \hat{\xi}_{1}$ are omitted as they produce zero result in the operation $(\hat{\xi} \times \hat{b} \mathbb{T})$ in eqns. (11) and (A2). The vector $(\hat{\xi} \times \hat{b} \mathbb{T})$ then involves products such as $\hat{\xi}_{1} \times\left[\hat{b}_{1}\left(\hat{\xi}_{2} \otimes \hat{\xi}_{2}\right)\right], \hat{\xi}_{1} \times\left[\hat{b}_{1}\left(\hat{\xi}_{1} \otimes \hat{\xi}_{2}\right)\right]$, etc., which can be worked out by expressing the vectors involved in an orthogonal basis such as $\left\{\hat{e}_{3}\right.$, 
$\left.\hat{\xi}_{1}, \hat{e}_{3} \times \hat{\xi}_{1}\right\}$, to give:

$$
\begin{aligned}
\hat{\xi}_{1} \times \hat{b}_{1} \mathbb{T} & =\frac{\left(b_{1 s} \cos \theta+b_{1 e} \sin \theta\right)}{b_{1}} \hat{e}_{3} \sin \theta\left(\frac{\cos ^{2} \theta}{\sin ^{4} \theta} \frac{\partial^{2} R}{\partial x^{2}}+2 \frac{\cos \theta}{\sin ^{4} \theta} \frac{\partial^{2} R}{\partial x \partial y}+\frac{1}{\sin ^{4} \theta} \frac{\partial^{2} R}{\partial y^{2}}\right) \\
& -\frac{b_{1 s}}{b_{1}} \hat{e}_{3} \sin \theta\left(\frac{\cos \theta}{\sin ^{4} \theta} \frac{\partial^{2} R}{\partial x^{2}}+\frac{1+\cos ^{2} \theta}{\sin ^{4} \theta} \frac{\partial^{2} R}{\partial x \partial y}+\frac{\cos \theta}{\sin ^{4} \theta} \frac{\partial^{2} R}{\partial y^{2}}\right) \\
& -\left[\frac{b_{1 n}}{b_{1}} \hat{e}_{3} \sin \theta-\frac{\left(b_{1 s} \cos \theta+b_{1 e} \sin \theta\right)}{b_{1}}\left(\hat{e}_{3} \times \hat{\xi}_{1}\right)\right]\left(\frac{\cos \theta}{\sin ^{2} \theta} \frac{\partial^{2} R}{\partial x \partial z}+\frac{1}{\sin ^{2} \theta} \frac{\partial^{2} R}{\partial y \partial z}\right) \\
& -\frac{b_{1 s}}{b_{1}}\left(\hat{e}_{3} \times \hat{\xi}_{1}\right)\left(\frac{1}{\sin ^{2} \theta} \frac{\partial^{2} R}{\partial x \partial z}+\frac{\cos \theta}{\sin ^{2} \theta} \frac{\partial^{2} R}{\partial y \partial z}\right)-\frac{b_{1 n}}{b_{1}}\left(\hat{e}_{3} \times \hat{\xi}_{1}\right) \frac{\partial^{2} R}{\partial z^{2}}
\end{aligned}
$$

With this, the kernels $g(\vec{R})$ and $h(\vec{R})$ for the glide and climb interactions in eqns. (11) and (A2), respectively, can be shown to be of the form:

$$
\begin{aligned}
& g(\vec{R})=\frac{\mu}{4 \pi b_{1}}\left\{\left(A_{1}^{g} x+A_{2}^{g} y+A_{3}^{g} z\right) / R^{3}+\left(C_{4}^{g} x^{3}+C_{5}^{g} y^{3}+C_{6}^{g} z^{3}+C_{7}^{g} x^{2} y+\right.\right. \\
& \left.\left.C_{8}^{g} x^{2} z+C_{9}^{g} y^{2} x+C_{10}^{g} y^{2} z+C_{11}^{g} z^{2} x+C_{12}^{g} z^{2} y+C_{13}^{g} x y z\right) /\left[(1-v) R^{5}\right]\right\} ;
\end{aligned}
$$

$$
\begin{aligned}
& h(\vec{R})=\frac{\mu}{4 \pi b_{1}}\left\{\left(A_{1}^{c} x+A_{2}^{c} y+A_{3}^{c} z\right) / R^{3}+\left(C_{4}^{c} x^{3}+C_{5}^{c} y^{3}+C_{6}^{c} z^{3}+C_{7}^{c} x^{2} y+C_{8}^{c} x^{2} z+\right.\right. \\
& \left.\left.C_{9}^{c} y^{2} x+C_{10}^{c} y^{2} z+C_{11}^{c} z^{2} x+C_{12}^{c} z^{2} y+C_{13}^{c} x y z\right) /\left[(1-v) R^{5}\right]\right\}
\end{aligned}
$$

where $(x, y, z)$ now specifies $\vec{R}$ in the oblique coordinate system in Figure 1(a), and the coefficients are given below. In the following, the notations involved are

$$
\begin{gathered}
b_{s}=\vec{b} \cdot \hat{\xi} ; b_{n}=\vec{b} \cdot \hat{e}_{3} ; b_{e}=\vec{b} \cdot\left(\hat{e}_{3} \times \hat{\xi}\right) \\
\sin \theta=\left|\hat{\xi}_{1} \times \hat{\xi}_{2}\right| ; \cos \theta=\hat{\xi}_{1} \cdot \hat{\xi}_{2} \\
\cos \beta=\hat{\xi}_{2} \cdot \hat{n} ; \cos \gamma=\hat{e}_{3} \cdot \hat{n}
\end{gathered}
$$

If dislocation segment $d-1$ on which the force is calculated is glissile and non-screw, then $\vec{b}_{1} \cdot \hat{n}=0$, and

$$
\cos \gamma=b_{1 e} / \sqrt{b_{1 e}^{2}+b_{1 n}^{2}} ; \cos \beta=-\sin \theta b_{1 n} / \sqrt{b_{1 e}^{2}+b_{1 n}^{2}} .
$$

If $d-1$ is purely screw, then $b_{1 n}=b_{1 e}=0$, and $\hat{n}$ needs to be defined separately. Since only edge dislocations are susceptible to climb, the kernel $h(\vec{R})$ for climb is for the case $b_{1 s}=0$, and $\hat{n}=\left(\hat{\xi}_{1} \times \hat{b}_{1}\right)$. 
The coefficients in eqn. (A4) are:

$$
\begin{aligned}
& A_{1}^{g}=\left[-b_{2 s} b_{1 n} \sin \theta+2 b_{2 n} b_{1 e}-b_{2 e} b_{1 n} \cos \theta+b_{2 n} b_{1 s} \frac{\cos \theta}{\sin \theta}\right] \cos \beta \\
& -\left(b_{2 s} \sin \theta+b_{2 e} \cos \theta\right)\left(b_{1 s} \cos \theta+b_{1 e} \sin \theta\right) \cos \gamma \\
& A_{2}^{g}=\left[b_{2 n} b_{1 s} \frac{\left(\sin ^{2} \theta-\cos ^{2} \theta\right)}{\sin \theta}+b_{2 e} b_{1 n}-2 b_{2 n} b_{1 e} \cos \theta\right] \cos \beta \\
& +\left[b_{2 e}\left(b_{1 s} \cos \theta+b_{1 e} \sin \theta\right)\right] \cos \gamma \\
& A_{3}^{g}=\left[b_{2 s} b_{1 s} \frac{\left(\sin ^{2} \theta-\cos ^{2} \theta\right)}{\sin \theta}+2 b_{2 e} b_{1 e} \sin \theta+2 b_{2 e} b_{1 s} \cos \theta-2 b_{2 s} b_{1 e} \cos \theta\right] \cos \beta \\
& C_{4}^{g}=\left[b_{1 s} b_{2 e}+b_{1 n} b_{2 n} \sin \theta\right] \cos \gamma+b_{2 n}\left(-b_{1 s} \frac{\cos \theta}{\sin \theta}+b_{1 e}\right) \cos \beta \\
& C_{5}^{g}=\left[-b_{2 e}\left(b_{1 s} \cos \theta+b_{1 e} \sin \theta\right)\right] \cos \gamma \\
& +\left[b_{2 n} b_{1 s} \frac{\left(2 \cos ^{2} \theta-1\right)}{\sin \theta}+2 b_{2 n} b_{1 e} \cos \theta-b_{2 e} b_{1 n}\right] \cos \beta \\
& C_{6}^{g}=\left[b_{2 n} b_{1 s} \sin \theta-b_{2 n} b_{1 e} \cos \theta\right] \cos \gamma+\left[\frac{b_{2 e} b_{1 e}-b_{1 n} b_{2 n} \cos \theta}{\sin \theta}\right] \cos \beta \\
& C_{7}^{g}=\left[-2 b_{2 n} b_{1 n} \cos \theta \sin \theta-3 b_{2 e} b_{1 s} \cos \theta-b_{2 e} b_{1 e} \sin \theta\right] \cos \gamma \\
& +\left[b_{2 n} b_{1 s} \frac{\left(2+\cos ^{2} \theta\right)}{\sin \theta}-b_{2 e} b_{1 n}\right] \cos \beta \\
& C_{8}^{g}=\left[-b_{2 n} b_{1 e} \cos \theta+3 b_{1 n} b_{2 e}-2 b_{1 s} b_{2 n} \sin \theta\right] \cos \gamma+\left[\frac{b_{2 e} b_{1 e}-b_{1 n} b_{2 n} \cos \theta}{\sin \theta}\right] \cos \beta \\
& C_{9}^{g}=\left[b_{1 n} b_{2 n} \sin \theta+b_{1 s} b_{2 e}\left(1+2 \cos ^{2} \theta\right)+2 b_{1 e} b_{2 e} \sin \theta \cos \theta\right] \cos \gamma \\
& +\left[-b_{2 n} b_{1 s} \frac{\cos \theta}{\sin \theta}\left(2+\cos ^{2} \theta\right)-b_{2 n} b_{1 e}\left(2+\cos ^{2} \theta\right)+2 b_{2 e} b_{1 n} \cos \theta\right] \cos \beta \\
& C_{10}^{g}=\left[b_{2 n} b_{1 s} \sin \theta-b_{2 n} b_{1 e} \cos \theta+3 b_{1 n} b_{2 e}\right] \cos \gamma \\
& +\left[-b_{2 n} b_{1 n} \frac{\cos \theta}{\sin \theta}-3 b_{2 e} b_{1 s} \cos \theta-b_{2 e} b_{1 e} \frac{\left(2-3 \cos ^{2} \theta\right)}{\sin \theta}\right] \cos \beta
\end{aligned}
$$




$$
\begin{aligned}
C_{11}^{g}= & {\left[-2 b_{1 s} b_{2 e}-2 b_{1 n} b_{2 n} \sin \theta\right] \cos \gamma+\left[b_{2 n}\left(-b_{1 s} \frac{\cos \theta}{\sin \theta}+b_{1 e}\right)\right] \cos \beta } \\
C_{12}^{g}=[ & {\left[2 b_{2 e}\left(b_{1 s} \cos \theta+b_{1 e} \sin \theta\right)\right] \cos \gamma } \\
& +\left[-b_{2 n} b_{1 s} \frac{\left(1-2 \cos ^{2} \theta\right)}{\sin \theta}+2 b_{2 n} b_{1 e} \cos \theta+2 b_{2 e} b_{1 n}\right] \cos \beta \\
C_{13}^{g}= & {\left[-6 b_{2 e} b_{1 n} \cos \theta+b_{2 n} b_{1 s} \cos \theta \sin \theta+b_{2 n} b_{1 e}\left(2+\sin ^{2} \theta\right)\right] \cos \gamma } \\
& +\left[b_{2 n} b_{1 n} \frac{\left(2+\sin ^{2} \theta\right)}{\sin \theta}+3 b_{2 e} b_{1 s}-2 b_{2 e} b_{1 e} \frac{\cos \theta}{\sin \theta}\right] \cos \beta
\end{aligned}
$$

The coefficients in eqn. (A5) are:

$$
\begin{aligned}
A_{1}^{c}= & \cos \gamma \sin \theta\left[b_{2 s} b_{1 n} \sin \theta-2 b_{2 n} b_{1 e}+b_{2 e} b_{1 n} \cos \theta\right] \\
& -b_{1 e} \cos \beta\left(b_{2 s} \sin \theta+b_{2 e} \cos \theta\right) \\
A_{2}^{c}= & \cos \gamma \sin \theta\left[-b_{2 e} b_{1 n}+2 b_{2 n} b_{1 e} \cos \theta\right]+b_{1 e} b_{2 e} \cos \beta \\
A_{3}^{c}= & 2 \cos \gamma \sin \theta\left[-b_{2 e} b_{1 e} \sin \theta+b_{2 s} b_{1 e} \cos \theta\right] \\
C_{4}^{c}= & 2 b_{1} b_{2 n} \sin \theta+\left[-b_{2 n} b_{1 e} \sin \theta\right] \cos \gamma+\left[b_{1 n} b_{2 n}\right] \cos \beta \\
C_{5}^{c}= & {\left[-2 b_{2 n} b_{1 e} \cos \theta+b_{1 n} b_{2 e}\right] \sin \theta \cos \gamma+\left[-b_{2 e} b_{1 e}\right] \cos \beta } \\
C_{6}^{c}= & 2 b_{1} b_{2 e}+\left[b_{1 n} b_{2 n} \cos \theta-b_{2 e} b_{1 e}\right] \cos \gamma+\left[-b_{2 n} b_{1 e} \frac{\cos \theta}{\sin \theta}\right] \cos \beta \\
C_{7}^{c}= & -4 b_{1} b_{2 n} \sin \theta \cos \theta+\left[b_{1 n} b_{2 e} \sin \theta\right] \cos \gamma+\left[-2 b_{1 n} b_{2 n} \cos \theta-b_{2 e} b_{1 e}\right] \cos \beta \\
& +\left[b_{1 n} b_{2 n}+2 b_{2 e} b_{1 e} \cos \theta\right] \cos \beta \\
C_{8}^{c}= & 2 b_{1} b_{2 e}+\left[b_{1 n} b_{2 n} \cos \theta-b_{2 e} b_{1 e}\right] \cos \gamma+\left[-b_{2 n} b_{1 e} \frac{\cos \theta}{\sin \theta}+\frac{3 b_{1 n} b_{2 e}}{\sin \theta] \cos \beta}\right. \\
C_{10}^{c}= & 2 b_{1} b_{2 e}+\left[b_{1 n} b_{2 n} \cos \theta+b_{2 e} b_{1 e}\left(2-3 \cos { }^{2} \theta\right)\right] \cos \gamma \\
C_{9}^{c}= & 2 b_{1} b_{2 n} \sin \theta+\left[b_{2 n} b_{1 e}\left(2+\cos { }^{2} \theta\right)-2 b_{1 n} b_{2 e} \cos \theta\right] \sin \theta \cos \gamma \\
& \\
&
\end{aligned}
$$




$$
\begin{aligned}
+ & {\left[-b_{2 n} b_{1 e} \frac{\cos \theta}{\sin \theta}+\frac{3 b_{1 n} b_{2 e}}{\sin \theta}\right] \cos \beta } \\
C_{11}^{c}= & 2 b_{1} b_{2 n} \sin \theta+\left[-b_{2 n} b_{1 e} \sin \theta\right] \cos \gamma+\left[-2 b_{1 n} b_{2 n}\right] \cos \beta \\
C_{12}^{c}= & {\left[-2 b_{2 n} b_{1 e} \sin \theta \cos \theta-2 b_{1 n} b_{2 e} \sin \theta\right] \cos \gamma+\left[2 b_{1 e} b_{2 e}\right] \cos \beta } \\
C_{13}^{c}= & -4 b_{1} b_{2 e} \cos \theta+\left[b_{1 n} b_{2 n}\left(-2-\sin ^{2} \theta\right)+2 b_{2 e} b_{1 e} \cos \theta\right] \cos \gamma \\
& +\left[b_{2 n} b_{1 e} \frac{\left(2+\sin ^{2} \theta\right)}{\sin \theta}-6 b_{1 n} b_{2 e} \frac{\cos \theta}{\sin \theta}\right] \cos \beta
\end{aligned}
$$

\section{Case (ii) - the two dislocation densities are parallel}

In this case, the oblique coordinate system in Figure 1(a) breaks down, and instead, a Cartesian system with mutually orthogonal unit basis vectors $\left\{\hat{\xi}_{2}, \hat{e}_{3} \times \hat{\xi}_{2}\right.$, $\left.\hat{e}_{3}\right\}$ is used, as explained by Hirth and Lothe (1992). Here, $\hat{\xi}_{2}$ points along the dislocation segment $d-2$, and segment $d-1$ along $\hat{\xi}_{1}$ is now parallel to the $\hat{\xi}_{2}$ axis, where $\left(\hat{\xi}_{1} \cdot \hat{\xi}_{2}\right)$ is \pm 1 depending on whether the two dislocations are parallel or anti-parallel. As before, $(x, y, z)$ represent the relative coordinates of $d-1$ from $d-2$ in the coordinate system $\left\{\hat{\xi}_{2}, \hat{e}_{3} \times \hat{\xi}_{2}, \hat{e}_{3}\right\}$. In eqns. (11) and (A2),

$$
\vec{\nabla}=\hat{\xi}_{2}\left(\frac{\partial}{\partial x}\right)+\hat{e}_{3} \times \hat{\xi}_{2}\left(\frac{\partial}{\partial y}\right)+\hat{e}_{3}\left(\frac{\partial}{\partial z}\right), \quad \nabla^{2} R=2 / R,
$$

and

$$
\begin{array}{r}
\hat{b}_{1} \mathbb{T}=\hat{\xi}_{2}\left(\frac{b_{1 s}}{b_{1}} \frac{\partial^{2} R}{\partial x^{2}}+\frac{b_{1 e}}{b_{1}} \frac{\partial^{2} R}{\partial x \partial y}+\frac{b_{1 n}}{b_{1}} \frac{\partial^{2} R}{\partial x \partial z}\right) \\
+\hat{e}_{3} \times \hat{\xi}_{2}\left(\frac{b_{1 s}}{b_{1}} \frac{\partial^{2} R}{\partial x \partial y}+\frac{b_{1 e}}{b_{1}} \frac{\partial^{2} R}{\partial y^{2}}+\frac{b_{1 n}}{b_{1}} \frac{\partial^{2} R}{\partial y \partial z}\right) \\
+\hat{e}_{3}\left(\frac{b_{1 s}}{b_{1}} \frac{\partial^{2} R}{\partial x \partial z}+\frac{b_{1 e}}{b_{1}} \frac{\partial^{2} R}{\partial y \partial z}+\frac{b_{1 n}}{b_{1}} \frac{\partial^{2} R}{\partial z^{2}}\right)
\end{array}
$$

The forms in eqn. (A4) and (A5) still apply. As before, we define the following quantities:

$$
\begin{gathered}
b_{1 s}=\vec{b}_{1} \cdot \hat{\xi}_{1} ; b_{1 n}=\vec{b}_{1} \cdot \hat{e}_{3} ; b_{1 e}=\vec{b}_{1} \cdot\left(\hat{e}_{3} \times \hat{\xi}_{1}\right) ; \\
b_{2 s}=\vec{b}_{2} \cdot \hat{\xi}_{2} ; b_{2 n}=\vec{b}_{2} \cdot \hat{e}_{3} ; b_{2 e}=\vec{b}_{2} \cdot\left(\hat{e}_{3} \times \hat{\xi}_{2}\right)
\end{gathered}
$$

In the following, we set $\hat{e}_{3}$ to be the slip plane normal $\hat{n}$ for dislocation segment $d-1$. 
The coefficients in eqn. (A4) are:

$$
\begin{aligned}
& A_{1}^{g}=\left(\hat{\xi}_{1} \cdot \hat{\xi}_{2}\right)\left[-b_{1 s} b_{2 e}\right] \\
& A_{2}^{g}=\left(\hat{\xi}_{1} \cdot \hat{\xi}_{2}\right)\left[b_{1 s} b_{2 s}\right] \\
& A_{3}^{g}=0 \\
& C_{4}^{g}=\left[b_{1 s} b_{2 e}\right]\left(\hat{\xi}_{1} \cdot \hat{\xi}_{2}\right) \\
& C_{5}^{g}=\left[-b_{1 n} b_{2 n}+b_{1 e} b_{2 e}\right]\left(\hat{\xi}_{1} \cdot \hat{\xi}_{2}\right) \\
& C_{6}^{g}=\left[-b_{1 e} b_{2 n}\right]\left(\hat{\xi}_{1} \cdot \hat{\xi}_{2}\right) \\
& C_{7}^{g}=\left[-b_{1 n} b_{2 n}+b_{1 e} b_{2 e}\right]\left(\hat{\xi}_{1} \cdot \hat{\xi}_{2}\right) \\
& C_{8}^{g}=\left[-b_{1 e} b_{2 n}+3 b_{1 n} b_{2 e}\right]\left(\hat{\xi}_{1} \cdot \hat{\xi}_{2}\right) \\
& C_{9}^{g}=\left[b_{1 s} b_{2 e}\right]\left(\hat{\xi}_{1} \cdot \hat{\xi}_{2}\right) \\
& C_{10}^{g}=\left[3 b_{1 n} b_{2 e}+2 b_{1 e} b_{2 n}\right]\left(\hat{\xi}_{1} \cdot \hat{\xi}_{2}\right) \\
& C_{11}^{g}=\left[-2 b_{1 s} b_{2 e}\right]\left(\hat{\xi}_{1} \cdot \hat{\xi}_{2}\right) \\
& C_{12}^{g}=\left[2 b_{1 n} b_{2 n}-2 b_{1 e} b_{2 e}\right]\left(\hat{\xi}_{1} \cdot \hat{\xi}_{2}\right) \\
& C_{13}^{g}=\left[3 b_{1 s} b_{2 n}\right]\left(\hat{\xi}_{1} \cdot \hat{\xi}_{2}\right)
\end{aligned}
$$

For the coefficients in eqn. (A5), since only edge dislocations can climb, we set $b_{1 s}=b_{1 n}=0$. The coefficients become:

$$
\begin{aligned}
& A_{1}^{c}=A_{2}^{c}=A_{3}^{c}=C_{4}^{c}=0 \\
& C_{5}^{c}=\left[-2 b_{1} b_{2 n}\right]\left(\hat{\xi}_{1} \cdot \hat{\xi}_{2}\right) \\
& C_{6}^{c}=\left[2 b_{1} b_{2 e}-b_{1 e} b_{2 e}\right]\left(\hat{\xi}_{1} \cdot \hat{\xi}_{2}\right)
\end{aligned}
$$


$C_{7}^{c}=\left[-2 b_{1} b_{2 n}+3 b_{1 e} b_{2 n}\right]\left(\hat{\xi}_{1} \cdot \hat{\xi}_{2}\right)$

$C_{8}^{c}=\left[2 b_{1} b_{2 e}-b_{1 e} b_{2 e}\right]\left(\hat{\xi}_{1} \cdot \hat{\xi}_{2}\right)$

$C_{9}^{c}=0$

$C_{10}^{c}=\left[2 b_{1} b_{2 e}+2 b_{1 e} b_{2 e}\right]\left(\hat{\xi}_{1} \cdot \hat{\xi}_{2}\right)$

$C_{11}^{c}=0$

$C_{12}^{c}=\left[-2 b_{1} b_{2 n}+3 b_{1 e} b_{2 n}\right]\left(\hat{\xi}_{1} \cdot \hat{\xi}_{2}\right)$

$C_{13}^{c}=0$

In these expressions, the term $\left(\hat{\xi}_{1} \cdot \hat{\xi}_{2}\right)$ represents the proper behavior of dipole interactions, and is needed when the interaction force calculated based on the coordinate system $\left\{\hat{\xi}_{2}, \hat{e}_{3} \times \hat{\xi}_{2}, \hat{e}_{3}\right\}$ for dislocation 2 is referred back to dislocation 1 as in eqns. (9) and (A2).

\section{Acknowledgment}

This work is supported by funding from Kingboard Endowed Professorship in Materials Engineering. 


\section{References}

Acharya, A., 2001. A model of crystal plasticity based on the theory of continuously distributed dislocations. J. Mech. Phys. Solids 49, 761-784.

Aghababaei, R., Joshi, S.P., 2013. Micromechanics of crystallographic size-effects in metal matrix composites induced by thermo-mechanical loading. Int. J. Plasticity 42, 65-82.

Aifantis, E.C., 1986. On the dynamical origin of dislocation patterns. Mater. Sci. Engg. $81,563-574$.

Akarapu, S., Zbib, H.M., Bahr, D.F., 2010. Analysis of heterogeneous deformation and dislocation dynamics in single crystal micropillars under compression. International Journal of Plasticity 26, 239-257.

Alankar, A., Mastorakos, I.N., Field, D.P., 2009. A dislocation-density-based 3D crystal plasticity model for pure aluminium. Acta Mater. 57, 5936-5946.

Alankar, A., Eisenlohr, P., Raabe, D., 2011. A dislocation density-based crystal plasticity constitutive model for prismatic slip in $\alpha$-titanium. Acta Mater. 59, 7003-7009.

Alankar, A., Field, D.P., Zbib, H.M., 2012. Explicit incorporation of cross-slip in a dislocation density-based crystal plasticity model. Phil. Mag. 92, 3084-3100.

Amodeo, R.J., Ghoneim, N.M., 1990. Dislocation dynamics .1. A proposed methodology for deformation micromechanics. Physical Review B 41, 6958-6967.

Arsenlis, A., Parks, D.M., 2002. Modeling the evolution of crystallographic dislocation density in crystal plasticity. J. Mech. Phys. Solids 50, 1979-2009.

Arsenlis, A., Parks, D.M., Becker, R., Bulatov, V.V., 2004. On the evolution of crystallographic dislocation density in non-homogeneously deforming crystals. J. Mech. Phys. Solids 52, 1213-1246.

Asaro, R.J., Rice, J.R., 1977. Strain localization in ductile single crystals. J. Mech. Phys. Solids 25, 309-338. 
Balluffi, R.W., 2012. Introduction to elasticity theory for crystal defects. Cambridge University Press, p.398.

Bargmann, S., Svendsen, B., Ekh, M., 2011. An extended crystal plasticity model for latent hardening in polycrystals. Comput. Mech. 48, 631-645.

Benzerga, A.A., 2009. Micro-pillar plasticity: 2.5D mesoscopic simulations. Journal of the Mechanics and Physics of Solids 57, 1459-1469.

Bertin, N., Capolungo, L., Beyerlein, I.J., 2013. Hybrid dislocation dynamics based strain hardening constitutive model. Int. J. Plasticity 49, 119-144.

Brenner, S.S., 1957. Plastic deformation of copper and silver whiskers. J. Appl. Phys. 28, 1023-1026.

Bulatov, V., Abraham, F., Kubin, L., Devincre, B., Yip, S., 1998. Dislocation junction and crystal plasticity: linking atomistic and mesoscale simulations. Nature 391, 669.

Bulatov, V.V., Cai, W., 2006. Computer Simulations of Dislocations. Oxford University Press, p. 196.

Busso, E.P., Meissonnier, F.T., O’Dowd, N.P., 2000. Gradient-dependent deformation of two-phase single crystals. J. Mech. Phys. Solids 48, 2333-2361.

Cheng, B., Leung, H.S., Ngan, A.H.W., 2014. Strength of metals under vibrations dislocation-density-function dynamics simulations". To appear in Phil. Mag.

Cordero, N.M., Forest, S., Busso, E.P., Berbenni, S., Cherkaoui, M., 2012. Grain size effects on plastic strain and dislocation density tensor fields in metal polycrystals. Comput. Mater. Sci. 52, 7-13.

Devincre, B., Condat, M., 1992. Model validation of a 3D simulation of dislocation dynamics : Discretization and line tension effects. Acta. Metall. 40, 2629-2637.

Devincre, B., Kubin, L.P., 1997. Mesoscopic simulations of dislocations and plasticity. Mater. Sci. Engg. A 234-236, 8-14. 
Devincre, B., Hoc, T., Kubin, L., 2008. Dislocation mean free paths and strain hardening of crystals. Science 320, 1745-1748.

Dou, R., Derby, B., 2009. A universal scaling law for the strength of metal micropillars and nanowires. Scripta Materialia 61, 524-527.

Dunne, F.P.E., Rugg, D., Walker, A., 2007. Lengthscale-dependent, elastically anisotropic, physically-based hcp crystal plasticity: Application to cold-dwell fatigue in Ti alloys. Int. J. Plasticity 23, 1061-1083.

Dunne, F.P.E., Kiwanuka, R., Wilkinson, A.J., 2012. Crystal plasticity analysis of micro-deformation, lattice rotation and geometrically necessary dislocation density. Proc. R. Soc. Lond. A, doi:10.1098/repa.2012.0050.

El-Awady, J.A., Wen, M., Ghoniem, N.M., 2009. The role of the weakest-link mechanism in controlling the plasticity of micropillars. Journal of the Mechanics and Physics of Solids 57, 32-50.

Engels, P., Ma, A., Hartmaier, A., 2012. Continuum simulation of the evolution of dislocation densities during nanoindentation. Int. J. Plasticity 38, 159-169.

Evers, L.P., Brekelmans, W.A.M., Geers, M.G.D., 2004. Non-local crystal plasticity model with intrinsic SSD and GND effects. J. Mech. Phys. Solids 52, 2379-2401.

Fivel, M.C., Canova, G.R., 1999. Developing rigorous boundary conditions to simulations of discrete dislocation dynamics. Modelling and Simulation in Materials Science and Engineering 7, 753-768.

Fourie, J.T., 1970. Sub-surface dislocation structure of deformed copper. Philosophical Magazine 21, 977-985.

Franciosi, P., Zaoui, A., 1982. Multislip in F.C.C. crystals: A theoretical approach compared with experimental data. Acta Metall. 30, 1627-1637.

Frick, C.P., Clark, B.G., Orso, S., Schneider, A.S., Arzt, E., 2008. Size effect on strength and strain hardening of small-scale [111] nickel compression pillars. Materials Science and Engineering: A 489, 319-329. 
Gómez-García, D., Devincre, B., Kubin, L., 2006. Dislocation Patterns and the Similitude Principle: 2.5D Mesoscale Simulations. Physical Review Letters 96.

Greer, J.R., De Hosson, J.T.M., 2011. Plasticity in small-sized metallic systems: Intrinsic versus extrinsic size effect. Progress in Materials Science 56, 654-724.

Greer, J., Nix, W., 2006. Nanoscale gold pillars strengthened through dislocation starvation. Physical Review B 73, 245410.

Greer, J.R., Oliver, W.C., Nix, W.D., 2005. Size dependence of mechanical properties of gold at the micron scale in the absence of strain gradients. Acta Materialia 53, 1821-1830.

Groma, I., 1997. Link between the microscopic and mesoscopic length-scale description of the collective behavior of dislocations. Phys. Rev. B 56, 5807 (1997).

Groma, I., Csikor, F.F., Zaiser, M., 2003. Spatial correlations and higher-order gradient terms in a continuum description of dislocation dynamics. Acta Mater. 51, 1271-1281.

Gu, R., Ngan, A.H.W., 2012. Effects of pre-straining and coating on plastic deformation of aluminum micro-pillars. Acta Mater. 60, 6102-6111.

Gu, R., Ngan, A.H.W., 2013. Dislocation arrangement in small crystal volumes determines power-law size dependence of yield strength. J. Mech. Phys. Solids 61, 1531-1542.

Hähner, P., 1996. A theory of dislocation cell formation based on stochastic dislocation dynamics. Acta Mater. 44, 2345-2352.

Hochrainer, T., Zaiser, M., Gumbsch, P., 2007. A three-dimensional continuum theory of dislocation systems: kinematics and mean-field formulation. Philos. Mag. 87, 1261-1282.

Hirschberger, C.B., Peerlings, R.H.J., Brekelmans, W.A.M., Geers, M.G.D., 2011. On the role of dislocation conservation in single-slip crystal plasticity. Modelling Simul. Mater. Sci. Eng. 19, 085002 (24pp). 
Hirth, J.P., Lothe, J., 1992. Theory of Dislocations. $2^{\text {nd }}$ Ed., Krieger Publishing Co., p. 125.

Huang, M., Zhao, L., Tong, J., 2012. Discrete dislocation dynamics modelling of mechanical deformation of nickel-based single crystal superalloys. International Journal of Plasticity 28, 141-158.

Hosford Jr, W. F., Fleischer, R. L., \& Backofen, W. A., 1960. Tensile deformation of aluminum single crystals at low temperatures. Acta Metallurgica, 8, 187-199.

Jennings, A.T., Burek, M.J., Greer, J.R., 2010. Microstructure versus Size: Mechanical Properties of Electroplated Single Crystalline $\mathrm{Cu}$ Nanopillars. Physical Review Letters 104.

Jennings, A.T., Greer, J.R., 2011. Tensile deformation of electroplated copper nanopillars. Philosophical Magazine 91, 1108-1120.

Johnston, W.G., Gilman, J.J., 1959. Dislocation velocities, dislocation densities, and plastic flow in lithium fluoride crystals. J. Appl. Phys. 30, 129-144.

Kiener, D., Grosinger, W., Dehm, G., 2009. On the importance of sample compliance in uniaxial microtesting. Scripta Materialia 60, 148-151.

Kim, J.-Y., Greer, J.R., 2009. Tensile and compressive behavior of gold and molybdenum single crystals at the nano-scale. Acta Materialia 57, 5245-5253.

Kim, J.-Y., Jang, D., Greer, J.R., 2012. Crystallographic orientation and size dependence of tension-compression asymmetry in molybdenum nano-pillars. International Journal of Plasticity 28, 46-52.

Komanduri, R., Chandrasekaran, N., Raff, L.M., 2001. Molecular dynamics (MD) simulation of uniaxial tension of some single-crystal cubic metals at nanolevel. International Journal of Mechanical Sciences 43, 2237-2260.

Kubin, L., Devincre, B., Hoc, T., 2008. Modeling dislocation storage rates and mean free paths in face-centered cubic crystals. Acta Mater. 56, 6040-6049. 
Lee, M.G., Lim, H., Adams, B.L., Hirth, J.P., Wagoner, R.H., 2010. A dislocation density-based single crystal constitutive equation. Int. J. Plasticity 26, 925-938.

Lee, S.-W., Han, S.M., Nix, W.D., 2009. Uniaxial compression of fcc Au nanopillars on an $\mathrm{MgO}$ substrate: The effects of prestraining and annealing. Acta Materialia 57, 4404-4415.

Lee, S.-W., Nix, W.D., 2012. Size dependence of the yield strength of fcc and bcc metallic micropillars with diameters of a few micrometers. Philosophical Magazine 92, 1238-1260.

Li, D., Zbib, H., Sun, X., Khaleel, M., 2013. Predicting plastic flow and irradiation hardening of iron single crystal with mechanism-based continuum dislocation dynamics. Int. J. Plasticity, in press.

Liu, Z.L., Zhuang, Z., Liu, X.M., Zhao, X.C., Zhang, Z.H., 2011. A dislocation dynamics based higher-order crystal plasticity model and applications on confined thin-film plasticity. Int. J. Plasticity 27, 201-216.

Ma, A., Roters, F., Raabe, D., 2006. A dislocation density based constitutive model for crystal plasticity FEM including geometrically necessary dislocations. Acta Mater. 54, 2169-2179.

Madec, R., Devincre, B., Kubin, L. P. 2002. Simulation of dislocation patterns in multislip. Scripta Materialia 47, 689-95.

Motz, C., Weygand, D., Senger, J., Gumbsch, P., 2009. Initial dislocation structures in 3-D discrete dislocation dynamics and their influence on microscale plasticity. Acta Materialia 57, 1744-1754.

Ng, K.S., Ngan, A.H.W., 2008. Stochastic nature of plasticity of aluminum micro pillars. Acta Mater. 56, 1712-1720.

Ngan, A.H.W., 2005. Dislocation patterning - a statistical mechanics perspective. Scripta Mater. 52, 1005-1010.

Ohashi, T., 2005. Crystal plasticity analysis of dislocation emission from micro voids. Int. J. Plasticity 21, 2071-2088. 
Olmsted, D. L., Hector Jr, L. G., Curtin, W. A., Clifton, R. J., 2005. Atomistic simulations of dislocation mobility in $\mathrm{Al}, \mathrm{Ni}$ and $\mathrm{Al} / \mathrm{Mg}$ alloys. Modelling and Simulation in Materials Science and Engineering, 13, 371-388.

Orowan, E., 1940. Problems of plastic gliding. Proc. Phys. Soc. Lond. 52, 8-22.

Parthasarathy, T.A., Rao, S.I., Dimiduk, D.M., Uchic, M.D., Trinkle, D.R., 2007. Contribution to size effect of yield strength from the stochastics of dislocation source lengths in finite samples. Scripta Materialia 56, 313-316.

Petukhov, B.V., 2001. A theory of sharp yield point in low-dislocation crystals. Technical Physics 46, 1389-1395.

Pontes, J., Walgraef, D., Aifantis, E.C., 2006. On dislocation patterning: Multiple slip effects in the rate equation approach. Int. J. Plasticity 22, 1486-1505.

Prasad, G.V.S.S., Goerdeler, M., Gottstein, G., 2005. Work hardening model based on multiple dislocation densities. Mater. Sci. Engg. A 400-401, 231-233.

Puri, S., Das, A., Acharya, A., 2011. Mechanical response of multicrystalline thin films in mesoscale field dislocation mechanics. J. Mech. Phys. Solids 59, 2400-2417.

Roters, F., Raabe, D., Gottstein, G., 2000. Work hardening in heterogeneous alloys A microstructural approach based on three internal state variables. Acta Mater. 48, 4181-4189.

Rao, S., Dimiduk, D., Parthasarathy, T., Uchic, M., Tang, M., Woodward, C., 2008. Athermal mechanisms of size-dependent crystal flow gleaned from three-dimensional discrete dislocation simulations. Acta Mater. 56, 3245-3259.

Sandfeld, S., Hochrainer, T., Gumbsch, P., Zaiser, M., 2010. Numerical implementation of a 3D continuum theory of dislocation dynamics and application to micro-bending. Phil. Mag. 90, 3697-3728.

Sandfeld, S., Hochrainer, T., Zaiser, M., Gumbsch, P., 2011. Continuum modeling of dislocation plasticity: theory, numerical implementation, and validation by discrete dislocation simulations. J. Mater. Res. 26, 623-632. 
Schneider, A.S., Kiener, D., Yakacki, C.M., Maier, H.J., Gruber, P.A., Tamura, N., Kunz, M., Minor, A.M., Frick, C.P., 2013. Influence of bulk pre-straining on the size effect in nickel compression pillars. Materials Science and Engineering: A 559, $147-158$.

Shan, Z.W., Mishra, R.K., Syed Asif, S.A., Warren, O.L., Minor, A.M., 2008. Mechanical annealing and source-limited deformation in submicrometre-diameter $\mathrm{Ni}$ crystals. Nature materials 7, 115-119.

Shanthraj, P., Zikry, M.A., 2012. Dislocation-density mechanisms for void interactions in crystalline materials. Int. J. Plasticity 34, 154-163.

Taupin, V., Varadhan, S., Fressengeas, C., Beaudoin, A.J., 2008. Directionality of yield point in strain-aged steels: the role of polar dislocations. Acta Mater. 56, 3002-3010.

Taylor, G.I., 1934. The mechanism of plastic deformation of crystals. Part I theoretical. Proc. R. Soc. London A 145, 362-387.

Uchic, M.D., Dimiduk, D.M., Florando, J.N., Nix, W.D., 2004. Sample dimensions influence strength and crystal plasticity. Science 305, 986-989.

Verdier, M., Fivel, M., Groma, I., 1998. Mesoscopic scale simulation of dislocation dynamics in fcc metals: Principles and applications. Modell. Simul. Mater. Sci. Engg. $6,755-770$.

Vinogradov, V., Yasnikov, I.S., Estrin, Y., 2012. Evolution of fractal structures in dislocation ensembles during plastic deformation. Phys. Rev. Lett. 108, 205504-1-5.

Vitek, V., Perrin, R.C., Bowen, D.K., 1970. Core structure of $1 / 2<111\rangle$ screw dislocations in bec crystals. Philos. Mag. 21, 1049-1073.

Vitek, V., 1974. Theory of core structures of dislocations in body-centered cubic metals. Crystal Lattice Defects 5, 1-34.

Volkert, C.A., Lilleodden, E.T., 2006. Size effects in the deformation of sub-micron Au columns. Philosophical Magazine 86, 5567-5579. 
Walgraef, D., Aifantis, E.C., 1985. Dislocation patterning in fatigued metals as a result of dynamical instabilities. J. Appl. Phys. 58, 688-691.

Watanabe I., Setoyama, D., Iwata, N., Nakahishi, K., 2010. Characterization of yielding behavior of polycrystalline metals with single crystal plasticity based on representative characteristic length. Int. J. Plasticity 26, 570-585.

Wesseling, P., 2001. Principles of Computational Fluid Dynamics, Springer-Verlag, p. 381.

Xu, S., Guo, Y., Ngan, A.H.W., 2013. A molecular dynamics study on the orientation, size, and dislocation confinement effects on the plastic deformation of Al nanopillars. International Journal of Plasticity 43, 116-127.

Yamakov, V., Wolf, D., Phillpot, S.R., Mukherjee, A.K., Gleiter, H., 2002. Dislocation processes in the deformation of nanocrystalline aluminium by molecular-dynamics simulation. Nature materials 1, 45-48.

Yonenaga, I., Sumino, K., 1978. Dislocation dynamics in the plastic deformation of silicon crystals I. Experiments. Phys. Stat. Sol. (a) 50, 685-693.

Yefimov, S. and Van der Giessen, E., 2005. Multiple slip in a strain-gradient plasticity model motivated by a statistical-mechanics description of dislocations. Int. J. Solids Strut. 42, 3375-3394.

Zaiser, M., Nikitas, M, Hochrainer, T., Aifantis, E.C., 2007. Modelling size effects using 3D density-based dislocation dynamics. Phil. Mag. 87, 1283-1306.

Zikanov, O., 2010. Essential Computational Fluid Dynamics. John Wiley \& Sons.

Zhou, C., Biner, S.B., LeSar, R., 2010. Discrete dislocation dynamics simulations of plasticity at small scales. Acta Materialia 58, 1565-1577.

Zhou, Z., Sun, Y., 2004. Evolution of dislocation patterns and its application in prediction of flow stress. Chinese Sci. Bulletin 49, 1527-1531. 
Table 1 - Parameters for the simulation example in section 3.5

\begin{tabular}{|l|c|}
\hline \multicolumn{1}{|c|}{ Parameters } & Values \\
\hline Poisson's ratio & 0.3 \\
\hline $\begin{array}{l}\text { Crystal dimensions (with periodic boundary } \\
\text { conditions), Fig. 7(a) }\end{array}$ & $50(x) \times 50(y) \times 5(z)$ cells \\
\hline Cell size $\Delta x, \Delta y, \Delta z$ section 3.3 & $200 b, 200 b, 200 b$ \\
\hline Capture radius of interaction force $R_{c}$, eqn. (18) & $400 b$ \\
\hline Radius of annihilation $R_{\text {ann }}$, eqn. (23) & $200 b$ \\
\hline Capture radius for annihilation of edge $R_{e}$, eqn. (23) & $200 b$ \\
\hline Capture radius for annihilation of screw $R_{s}$, eqn. (23) & $200 b$ \\
\hline
\end{tabular}

Table 2 - Proof stress of micro-specimens with and without elastic interactions between dislocations.

\begin{tabular}{|c|c|c|c|}
\hline $\begin{array}{c}\text { Specimen } \\
\text { size }\end{array}$ & $\begin{array}{c}\sigma_{0.2 \%} / \mu \text { with } \\
\text { interaction stress } \\
\left(\times 10^{-3}\right)\end{array}$ & $\begin{array}{c}\sigma_{0.2 \%} / \mu \text { without } \\
\text { interaction stress } \\
\left(\times 10^{-3}\right)\end{array}$ & $\begin{array}{c}\% \text { change without } \\
\text { interaction stress }\end{array}$ \\
\hline $1000 \mathrm{~b}$ & 3.35 & 4.39 & 30.93 \\
\hline $2000 \mathrm{~b}$ & 2.02 & 2.64 & 30.79 \\
\hline $4000 \mathrm{~b}$ & 1.40 & 1.65 & 18.18 \\
\hline $6000 \mathrm{~b}$ & 1.20 & 1.36 & 13.31 \\
\hline $8000 \mathrm{~b}$ & 1.09 & 1.20 & 10.30 \\
\hline $16000 \mathrm{~b}$ & 1.04 & 9.94 & -4.44 \\
\hline
\end{tabular}




\section{Figure Captions}

Figure 1 - Elastic interaction between (a) two dislocation segments, and (b) a dislocation segment at $\vec{r}$ and a density of dislocations at $\vec{r}^{\prime}$.

Figure 2 - Edge-screw idealization of slip systems.

Figure 3 - Production of new dislocations in the edge-screw idealization by (a) expansion of a rectangular loop, (b) bow-out, and (c) trailing dislocations.

Figure 4 - Cross-slip in the edge-screw idealization.

Figure 5 - Dislocation generation in FVM cells.

Figure 6 - Dislocation annihilation in FVM cells.

Figure 7 - (a) Schematic of the simulation block. (b) Simulated stress-strain behavior of single crystal aluminum under uniaxial tensile loading. The dashed lines are experimental results from Hosford et al. (1960) and the solid lines are simulation results.

Figure 8 - (a): Strain evolution of the specimen under tensile stress along $\langle 100\rangle$ lattice direction. (b) The stress-strain curve. (c) Dislocation density evolution for the four slip systems. (d) Evolution of the Taylor resistance and elastic interaction stress between dislocations. (Stresses are normalized by shear modulus $\mu$.) (e to $\mathrm{h}$ ): Dislocation density distribution evolution. The density maps shown are sections near the middle of the cell along the $z$ direction. Dislocation density scale is in units of $\mathrm{m}^{-2}$. Strain along the tensile direction $\left(\varepsilon_{x x}\right)$ is specified under each snapshot.

Figure 9 - Simulated stress-strain curves for various specimen sizes loaded at a constant strain rate of $2.5 \times 10^{-3} \mathrm{~s}^{-1}$.

Figure 10 - Comparison of the present simulation results with data in the literature. The upper stress values of the sharp yield points in Figure 9 are used to convert into resolved shear strengths here with a Schmid factor of 0.408 assumed.

Figure 11 - Dislocation evolution of different specimen sizes with time.

Figure 12 - Evolution of dislocation-density contour plots as specimens of different sizes undergo deformation to different cumulative strains. Dislocation density in units of $\mathrm{m}^{-2}$.

Figure 13 - Effects of switching off mutual elastic interactions between dislocations. (a) Dislocation density vs time and (b) dislocation density plots at $0.2 \%$ strain of different specimen sizes with elastic interactions switched off. The density scale in (b) is the same as in Figure 12. 
Figure 14 - The Orowan strain as a compatible, pure-shear, component of the total plastic strain tensor due to movement of a dislocation, relative to the initial dislocation-free state. The remaining part of the plastic deformation tensor is due to the self-field of the dislocation and contains incompatibility. 


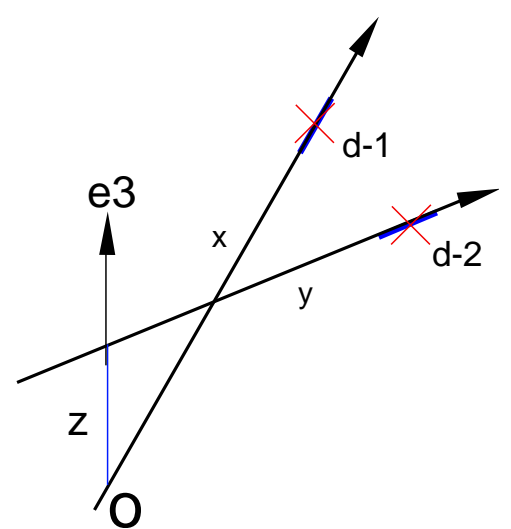

(a)

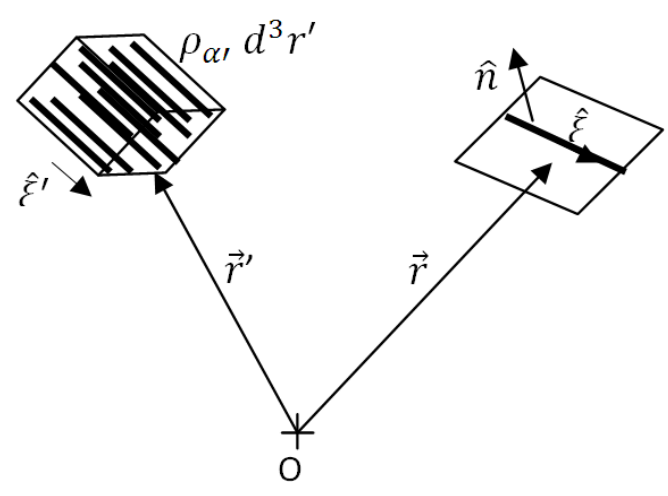

(b)

Figure 1 - Elastic interaction between (a) two dislocation segments, and (b) a dislocation segment at $\vec{r}$ and a density of dislocations at $\vec{r}^{\prime}$.

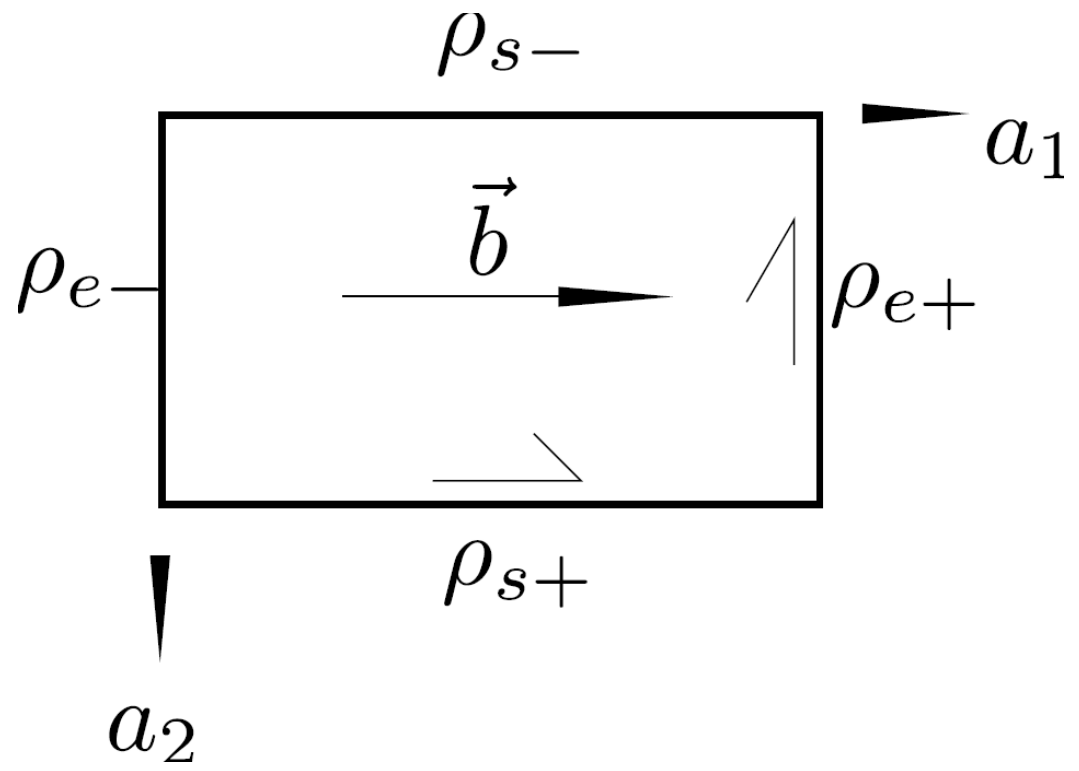

Figure 2 - Edge-screw idealization of slip systems. 


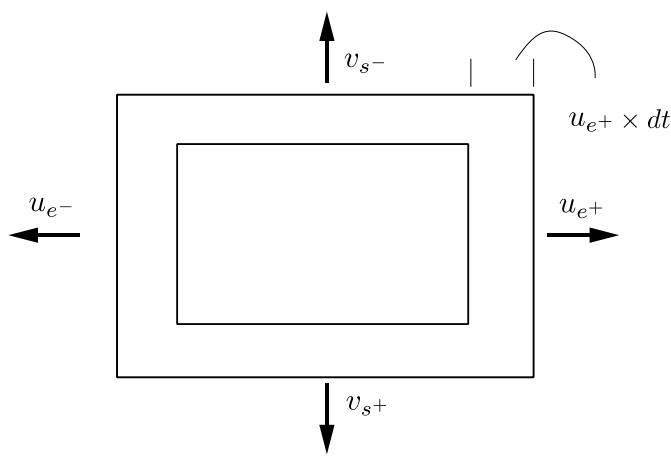

(a)

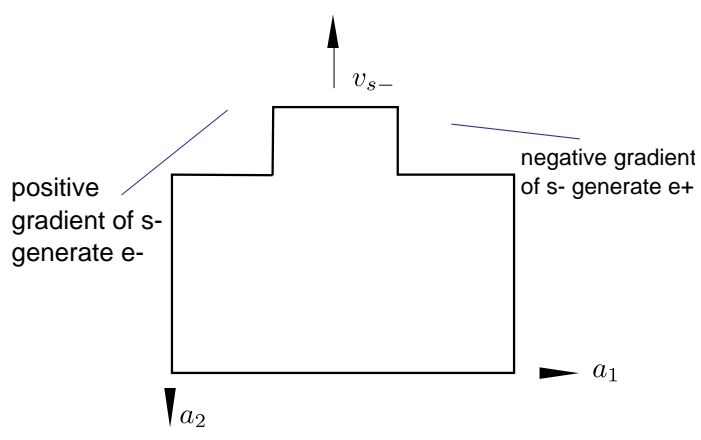

(b)

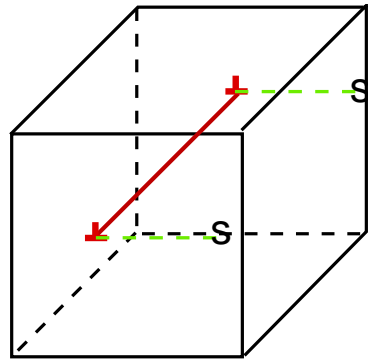

(c)

Figure 3 - Production of new dislocations in the edge-screw idealization by (a) expansion of a rectangular loop, (b) bow-out, and (c) trailing dislocations.

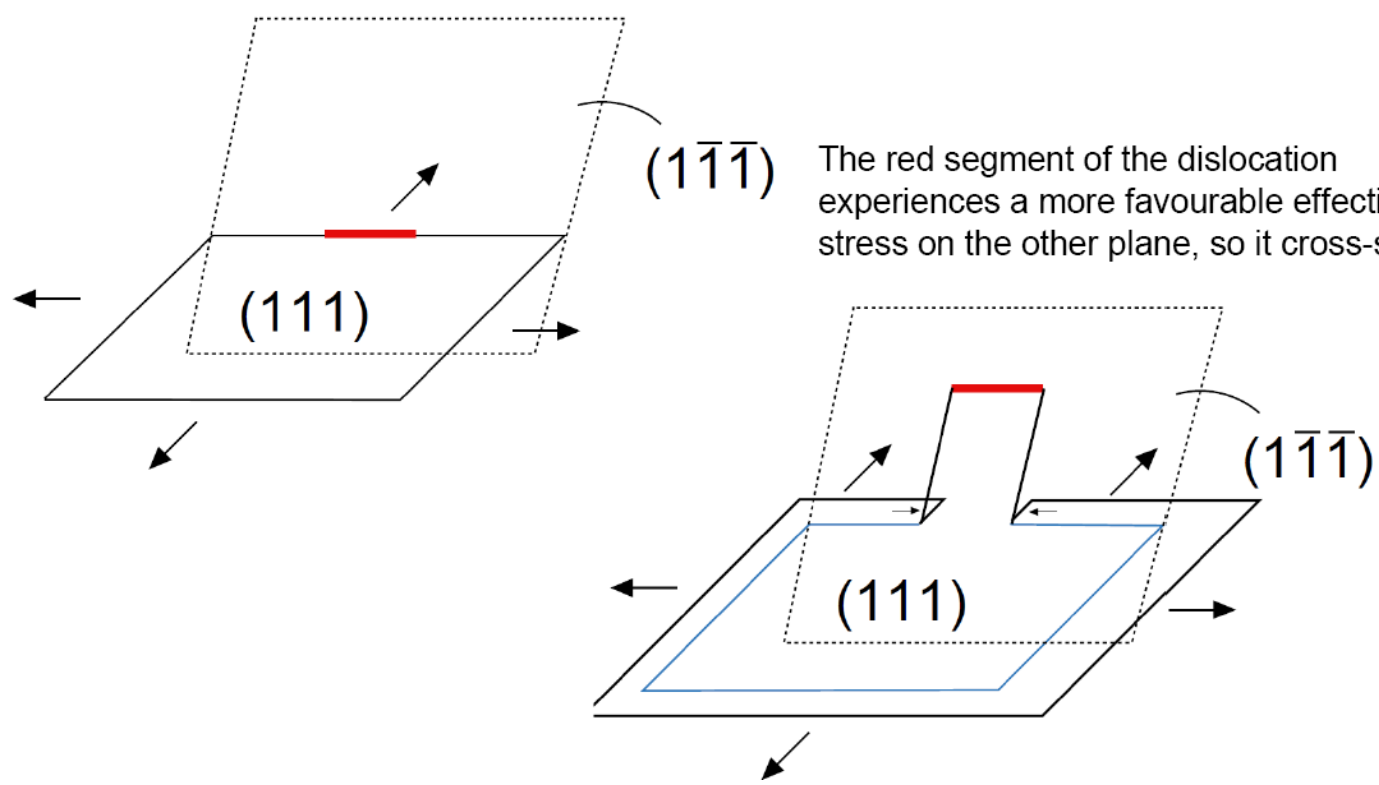

Figure 4 - Cross-slip in the edge-screw idealization. 


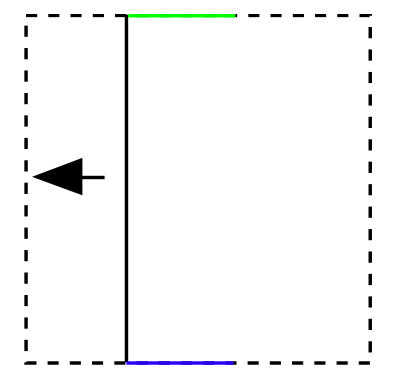

(a)

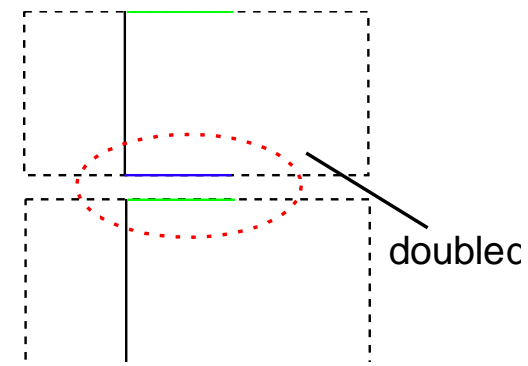

(b)

Figure 5 - Dislocation generation in FVM cells.

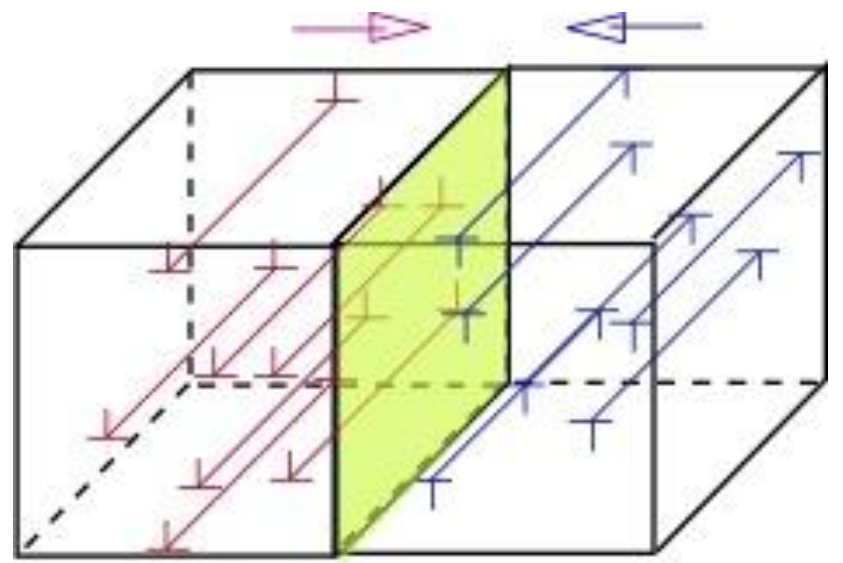

Figure 6 - Dislocation annihilation in FVM cells. 


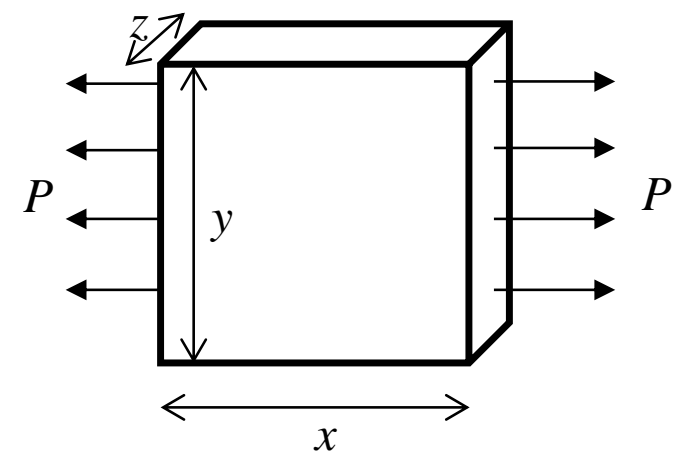

(a)

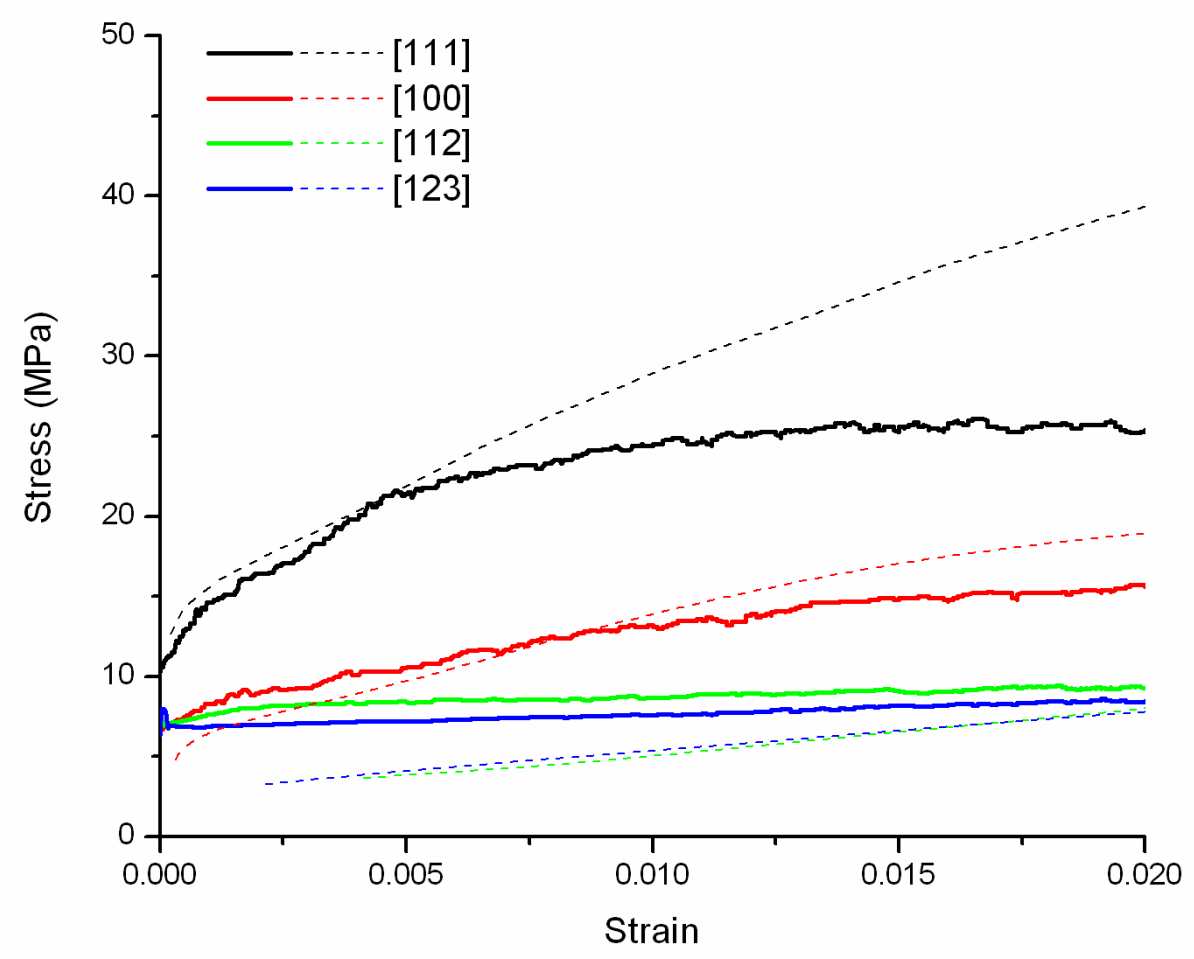

(b)

Figure 7 - (a) Schematic of the simulation block. (b) Simulated stress-strain behavior of single crystal aluminum under uniaxial tensile loading. The dashed lines are experimental results from Hosford et al. (1960) and the solid lines are simulation results. 


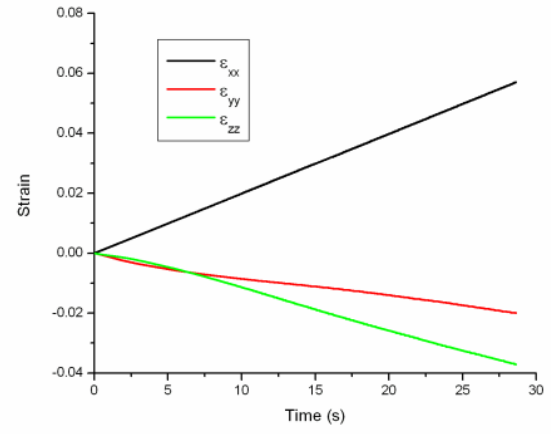

(a)

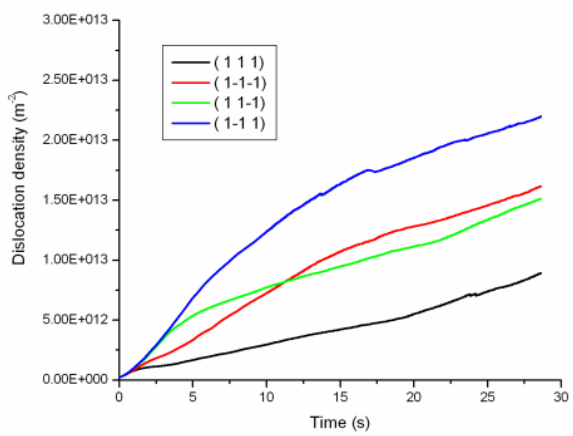

(c)

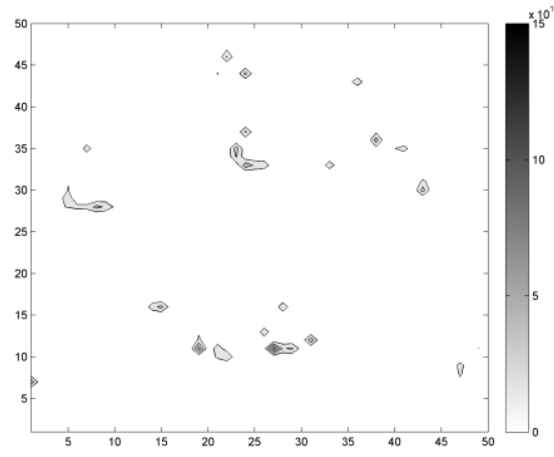

(e) Strain $=0.001$

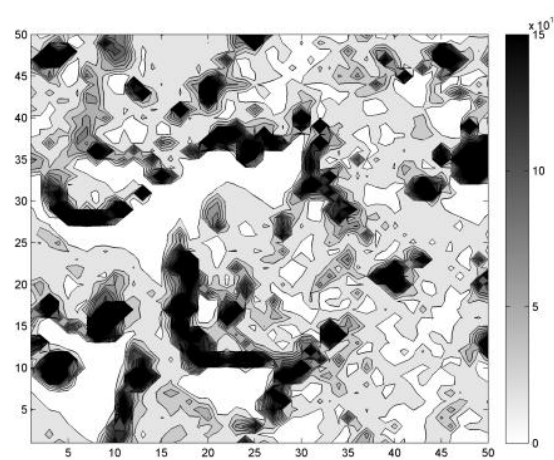

(g) Strain $=0.02$

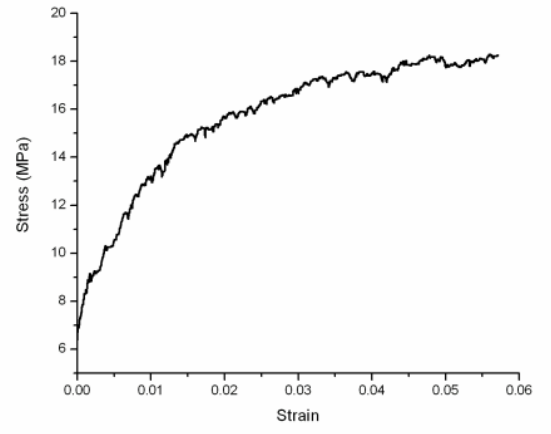

(b)

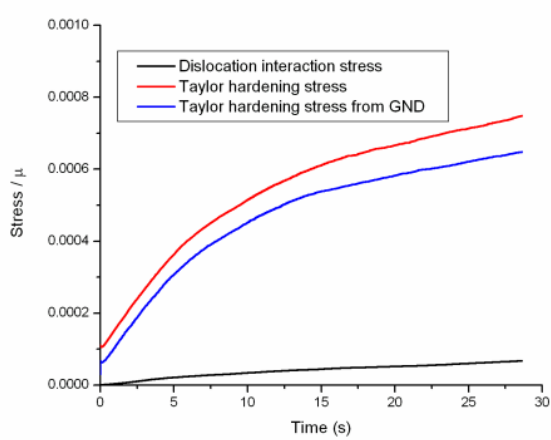

(d)

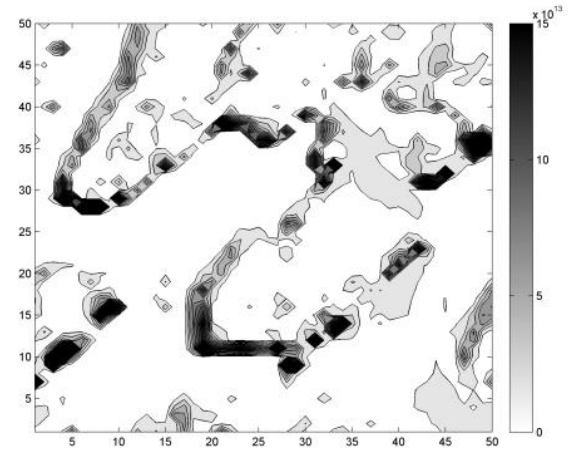

(f) Strain $=0.01$

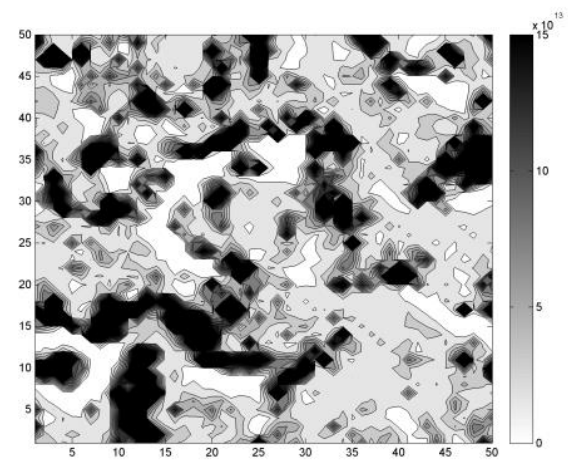

(h) Strain $=0.03$

Figure 8 - (a): Strain evolution of the specimen under tensile stress along $\langle 100\rangle$ 
lattice direction. (b) The stress-strain curve. (c) Dislocation density evolution for the four slip systems. (d) Evolution of the Taylor resistance and elastic interaction stress between dislocations. (Stresses are normalized by shear modulus $\mu$.) (e to $h$ ): Dislocation density distribution evolution. The density maps shown are sections near the middle of the cell along the $z$ direction. Dislocation density scale is in units of $\mathrm{m}^{-2}$. Strain along the tensile direction $\left(\varepsilon_{x x}\right)$ is specified under each snapshot. 


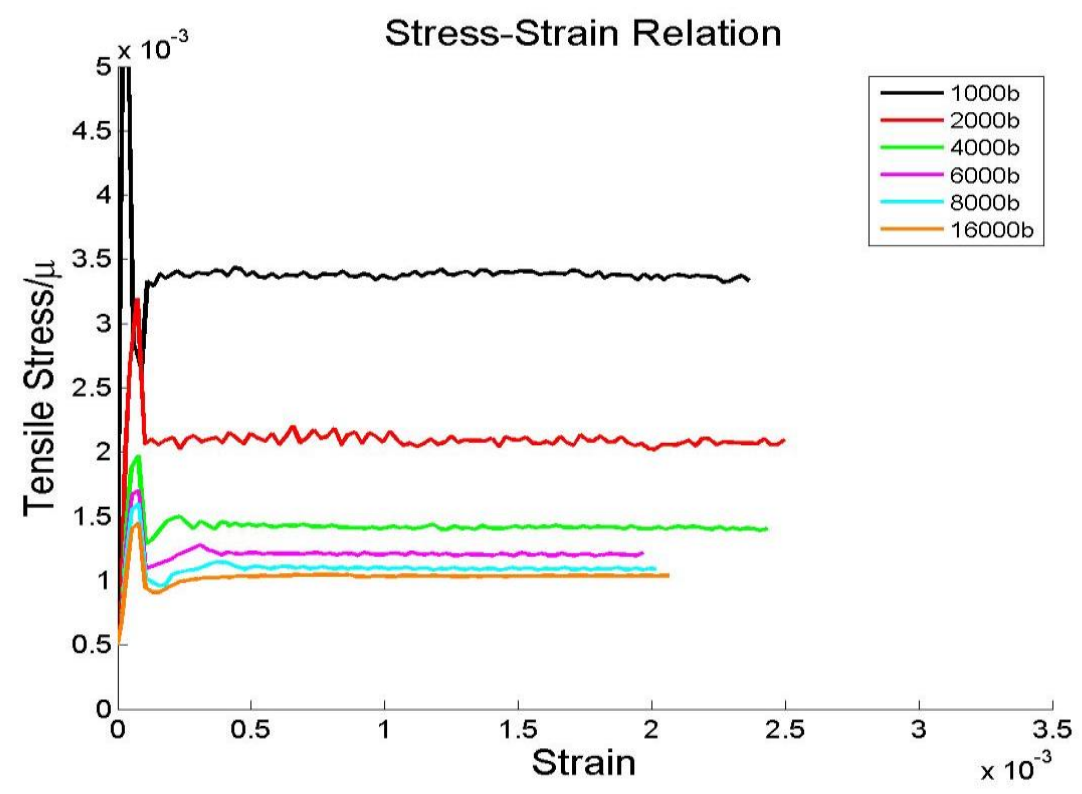

Figure 9 - Simulated stress-strain curves for various specimen sizes loaded at a constant strain rate of $2.5 \times 10^{-3} \mathrm{~s}^{-1}$.

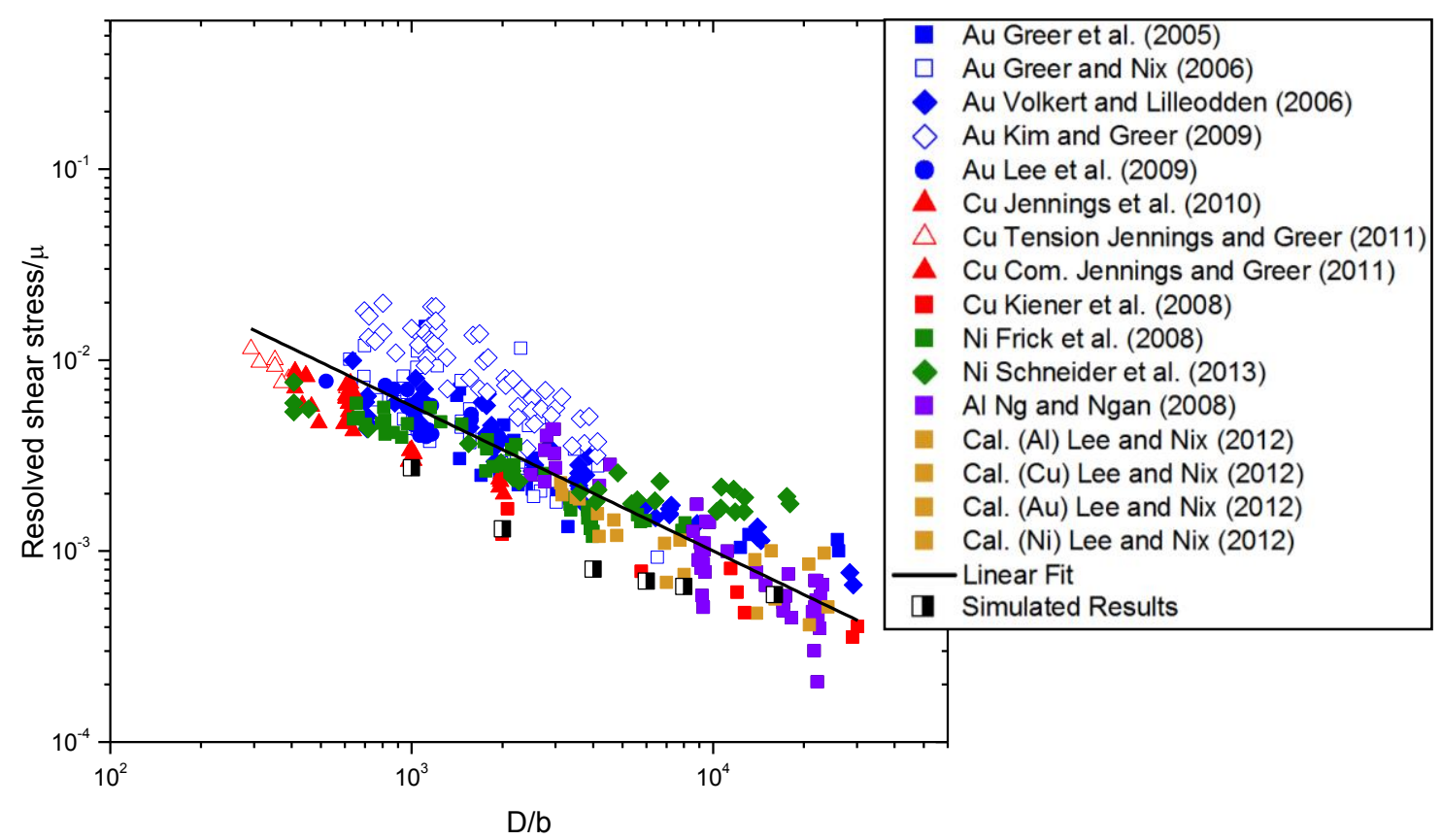

Figure 10 - Comparison of the present simulation results with data in the literature. The upper stress values of the sharp yield points in Figure 9 are used to convert into resolved shear strengths here with a Schmid factor of 0.408 assumed. 


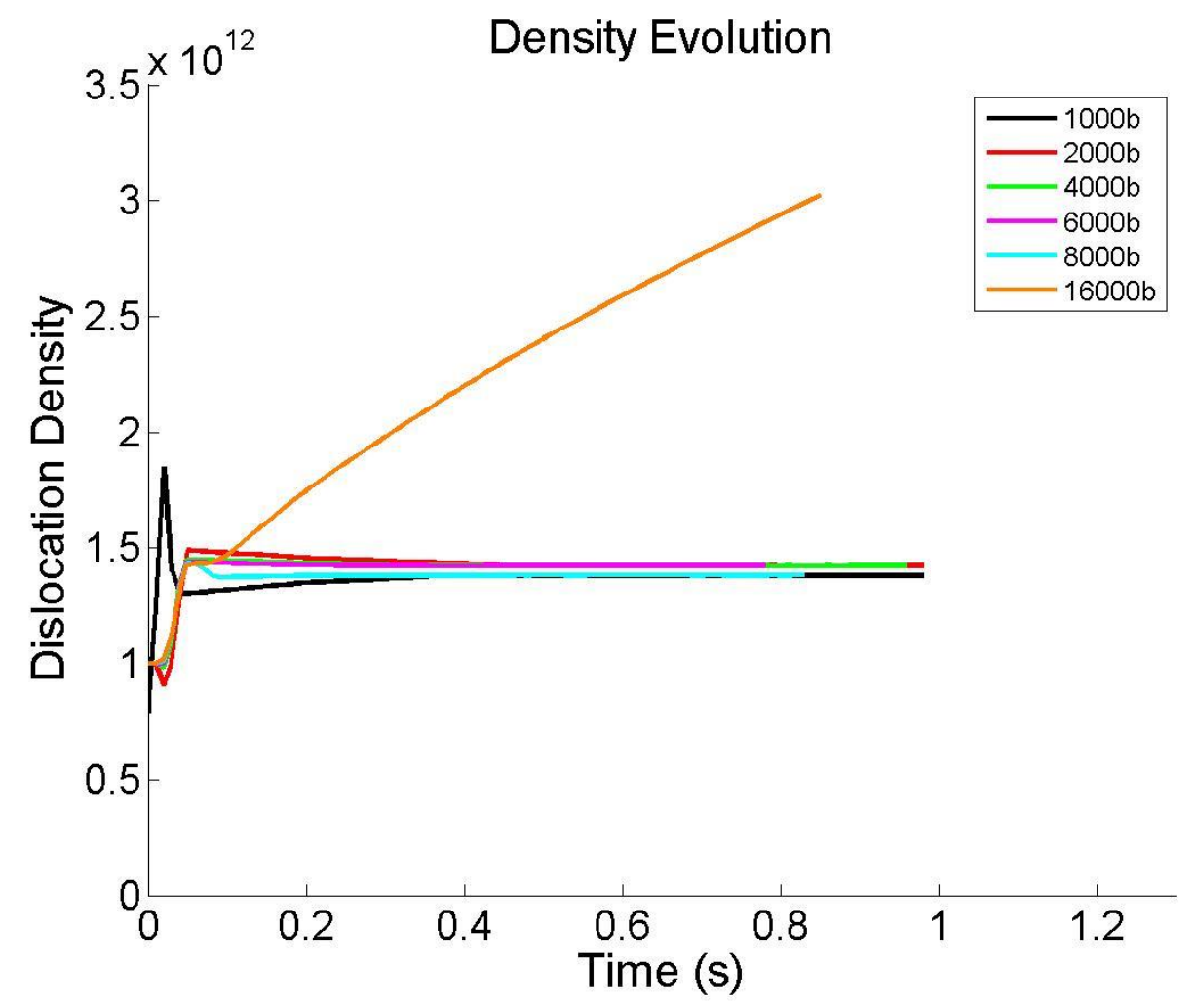

Figure 11 - Dislocation evolution of different specimen sizes with time.

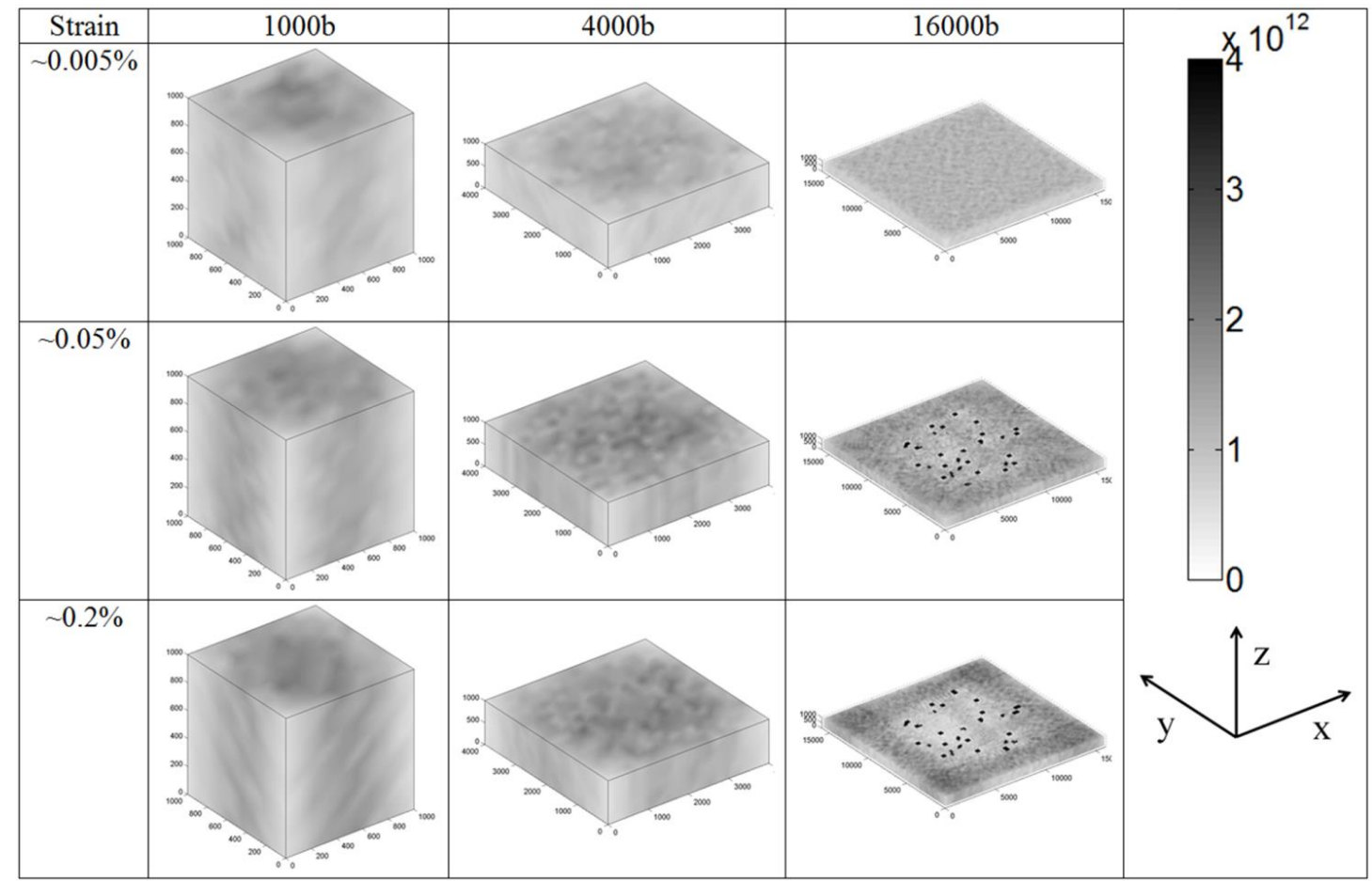

Figure 12 - Evolution of dislocation-density contour plots as specimens of different sizes undergo deformation to different cumulative strains. Dislocation density in units of $\mathrm{m}^{-2}$. 


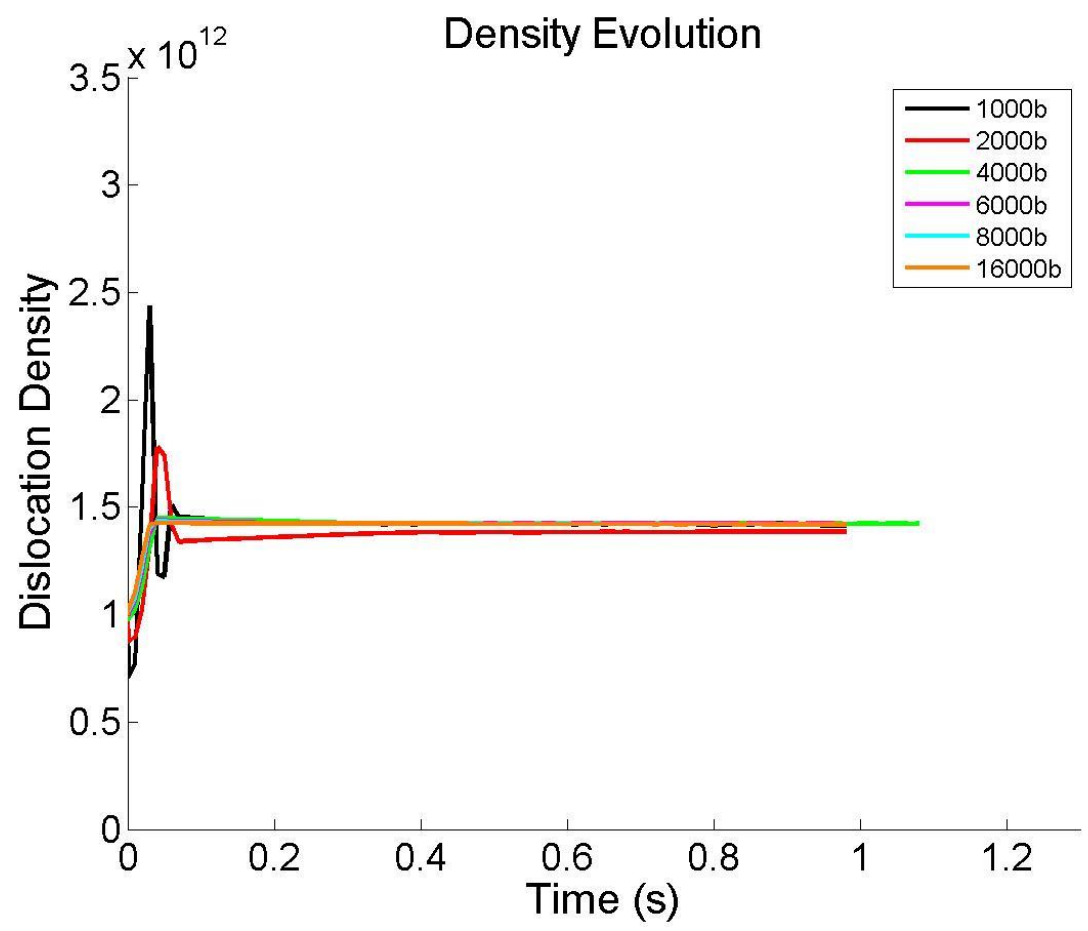

(a)

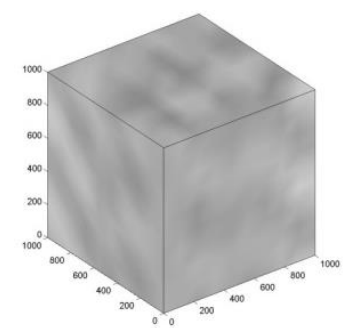

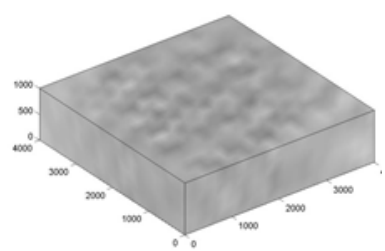

$4000 b$

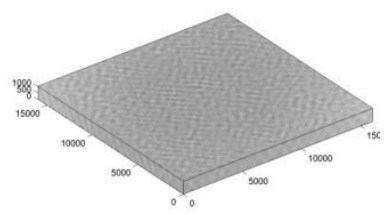

$16000 b$

(b)

Figure 13 - Effects of switching off mutual elastic interactions between dislocations. (a) Dislocation density vs time and (b) dislocation density plots at $0.2 \%$ strain of different specimen sizes with elastic interactions switched off. The density scale in (b) is the same as in Figure 12. 

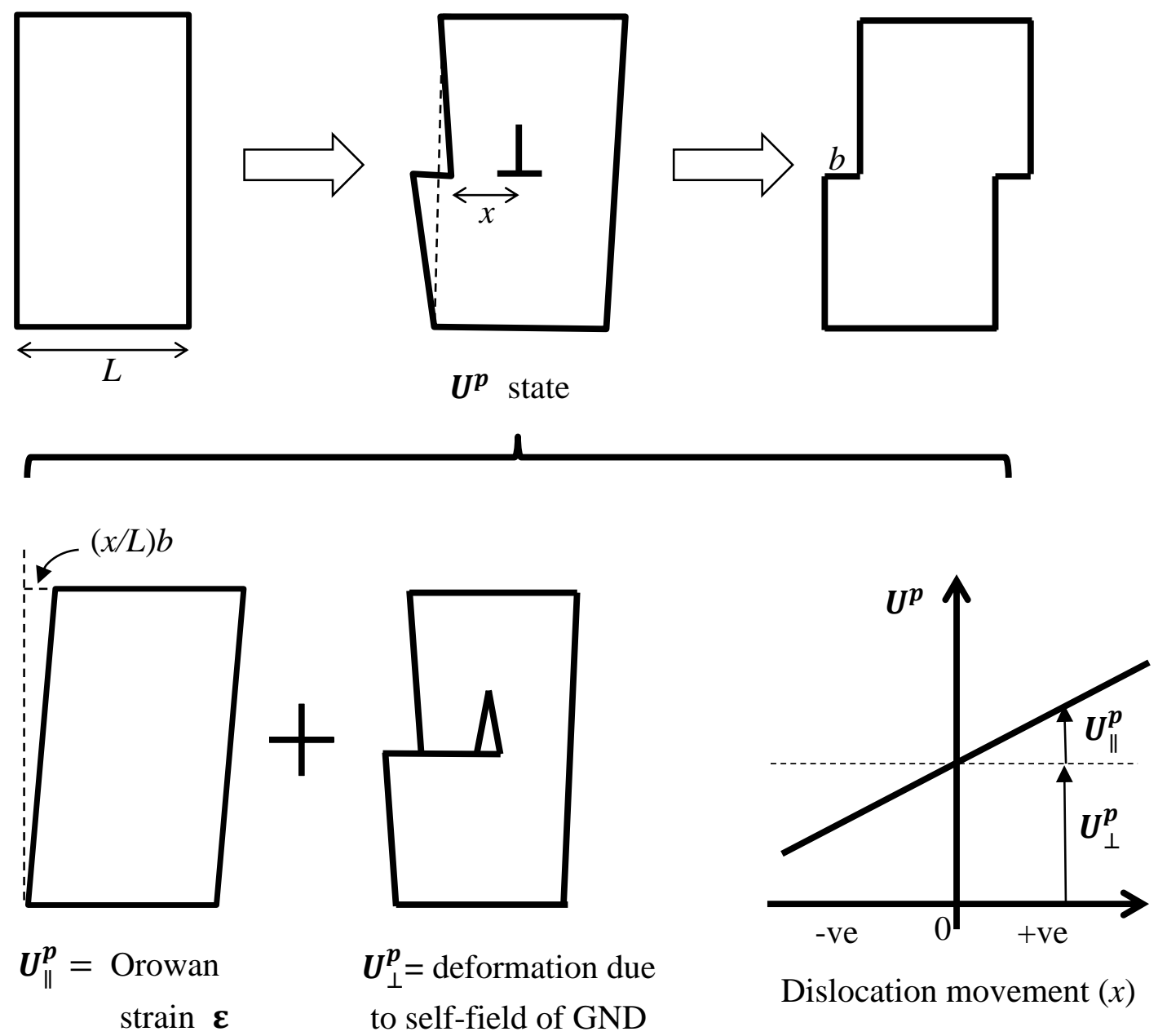

Dislocation movement $(x)$

Figure 14 - The Orowan strain as a compatible, pure-shear, component of the total plastic strain tensor due to movement of a dislocation, relative to the initial dislocation-free state. The remaining part of the plastic deformation tensor is due to the self-field of the dislocation and contains incompatibility. 